\title{
Androgen receptor signaling regulates the transcriptome of prostate cancer cells by modulating global alternative splicing
}

\author{
Kalpit Shah $\mathbb{D}^{1,6} \cdot$ Teresa Gagliano $\mathbb{D}^{2} \cdot$ Lisa Garland ${ }^{1} \cdot$ Timothy O'Hanlon $^{3} \cdot$ Daria Bortolotti ${ }^{4}$ - Valentina Gentili ${ }^{4}$. \\ Roberta Rizzo ${ }^{4}$. Georgios Giamas ${ }^{5} \cdot$ Michael Dean $\mathbb{1 0}^{1}$
}

Received: 20 February 2020 / Revised: 28 July 2020 / Accepted: 10 August 2020 / Published online: 20 August 2020

(c) The Author(s) 2020. This article is published with open access

\begin{abstract}
Androgen receptor (AR), is a transcription factor and a member of a hormone receptor superfamily. AR plays a vital role in the progression of prostate cancer and is a crucial target for therapeutic interventions. While the majority of advanced-stage prostate cancer patients will initially respond to the androgen deprivation, the disease often progresses to castrate-resistant prostate cancer (CRPC). Interestingly, CRPC tumors continue to depend on hyperactive AR signaling and will respond to potent second-line antiandrogen therapies, including bicalutamide (CASODEX ${ }^{\circ}$ ) and enzalutamide (XTANDI ${ }^{\circ}$. However, the progression-free survival rate for the CRPC patients on antiandrogen therapies is only 8-19 months. Hence, there is a need to understand the mechanisms underlying CRPC progression and eventual treatment resistance. Here, we have leveraged next-generation sequencing and newly developed analytical methodologies to evaluate the role of AR signaling in regulating the transcriptome of prostate cancer cells. The genomic and pharmacologic stimulation and inhibition of AR activity demonstrates that AR regulates alternative splicing within cancer-relevant genes. Furthermore, by integrating transcriptomic data from in vitro experiments and in prostate cancer patients, we found that a significant number of ARregulated splicing events are associated with tumor progression. For example, we found evidence for an inadvertent ARantagonist-mediated switch in $I D H 1$ and $P L 2 G 2 A$ isoform expression, which is associated with a decrease in overall survival of patients. Mechanistically, we discovered that the epithelial-specific splicing regulators (ESRP1 and ESRP2), flank many AR-regulated alternatively spliced exons. And, using 2D invasion assays, we show that the inhibition of ESRPs can suppress AR-antagonist-driven tumor invasion. Our work provides evidence for a new mechanism by which AR alters the transcriptome of prostate cancer cells by modulating alternative splicing. As such, our work has important implications for CRPC progression and development of resistance to treatment with bicalutamide and enzalutamide.
\end{abstract}

These authors contributed equally: Teresa Gagliano, Lisa Garland

Supplementary information The online version of this article (https:// doi.org/10.1038/s41388-020-01429-2) contains supplementary material, which is available to authorized users.

Kalpit Shah

kalpit.shah2016@gmail.com

$\triangle$ Michael Dean

michael.dean@nih.gov

1 Division of Cancer Epidemiology and Genetics, National Cancer Institute (NCI), National Institutes of Health (NIH), Bethesda, MD 20892, USA

2 Department of Medical Science, University of Udine, Udine, Italy

\section{Introduction}

Androgen receptor (AR) is a member of the superfamily of hormonal nuclear receptors [1]. In the absence of its ligand, AR is secured in the cytoplasm by heat-shock proteins [2]. Once exposed to the male hormone androgen, AR, becomes activated, and translocates to the nucleus, where it binds to

3 Cancer Genomics Research Laboratory, Frederick National Laboratory for Cancer Research, Bethesda, MD 20892, USA

4 Department of Chemical and Pharmaceutical Sciences, University of Ferrara, Ferrara, Italy

5 Department of Biochemistry and Biomedicine, School of Life Sciences, University of Sussex, Falmer, Brighton BN1 9QG, UK

6 Present address: Genentech, 1 DNA Way, South San Francisco, CA 94080, USA 
the androgen response elements (ARE) and initiate the transcriptional program [3-7]. Interestingly, activated AR molecules both enhance and suppress the expression of genes involved in prostate cancer progression [8-13]. This hormone-driven AR signaling is essential for development, differentiation, and normal functioning of the prostatic gland [14]. AR signaling, however, is hijacked in prostate tumors, driving disease progression. Therefore, the blockage of AR signaling through androgen deprivation continues to be the mainstay treatment of advanced-stage prostate cancer. While almost all patients with metastatic disease will initially respond to androgen-ablation therapies, the majority of patients will progress to a castrate-resistant stage [15-18].

Interestingly, studies employing xenograft prostate tumor models have shown that castrate-resistant prostate cancer (CRPC) tumors that emerge after androgen-ablation therapy, continue to express AR and AR-regulated genes [19]. Recent studies have argued that kinase-mediated hypersensitivity of AR [20-24] and efficient uptake of androgens may play a critical role in fueling CRPC tumors [25]. Thus, the treatment option for patients with non-metastatic or metastatic CRPC typically includes high-affinity antiandrogens like bicalutamide (CASODEX ${ }^{\circ}$ ) and enzalutamide (XTANDI ${ }^{\circ}$ ) [26-28]. Although in recent trials enzalutamide has shown improved efficacy in comparison to bicalutamide, the median time to PSA progression still suggests a limited benefit that lasts no more than 8-19 months [29]. In addition, in a few cases, an increase in metastasis of the disease was reported to be associated with the AR-antagonist's treatment regimen. Therefore, the search for the mechanism underlying CRPC, CRPC progression, and eventual treatment resistance would benefit patients who have exhausted all currently available treatment options. Toward this effort, the comprehensive understanding of AR functions continues to remain the center of focus.

The recent advent of high-throughput RNA sequencing and splicing microarrays has unveiled new layers of regulation of gene expression and highlighted the extreme complexity and versatility of the transcriptome. The majority of human genes encode multiple transcripts through the use of alternative promoters, alternative splicing (ASE), and alternative polyadenylation [30]. ASE is a mechanism that significantly expands the functional potential of the genome either by altering the usage of protein-coding transcripts, the ratio of coding to noncoding transcripts, or by allowing expression of isoforms with antagonistic functions from a single gene [31]. Multiple studies have found that ASE plays a critical role in cancer [32]. A recent comprehensive analysis of ASE across 32 cancer types from 8705 patients revealed that tumors have up to $30 \%$ more ASEs than normal tissues [33]. The steroid nuclear hormone receptors, including estrogen and progesterone receptors, are known to recruit regulators of ASE and modulate the transcriptome [34, 35]. However, whether modulation of AR signaling may alter transcriptome of prostate cancer cells via alternative splicing remains largely unexplored.

Herein, we hypothesized that modulation of AR signaling either during prostate cancer progression or in response to treatment with AR antagonists might dysregulate the transcriptome of prostate cancer cells by modulating ASE. We employed a multitude of genomic approaches including Affymetrix splicing array, whole transcriptome RNA-seq analysis, and RT-PCR to show that AR signaling regulates the transcriptome of prostate cancer cells by modulating ASE of a wide array of genes involved in regulating protein function. Furthermore, leveraging publicly available transcriptome data of primary-site samples from patients with prostate cancer at various stages of progression, we found a subset of AR-driven splicing events that are associated with progression of prostate cancer. Mechanistically, we found that epithelial splicing regulator proteins (ESRP1 and ESRP2) are the splicing factor through which AR may regulate splicing of pre-mRNA in prostate cancer cells. Interestingly, the inhibition of ESRPs suppressed ARantagonist-mediated increase in the invasion of prostate cancer cells. Collectively, we provide the evidence for a novel and critical mechanism of prostate cancer progression that is regulated by $\mathrm{AR}$ and that the treatment with $\mathrm{AR}$ antagonist may inadvertently promote invasion by dysregulating splicing of critical genes. Our analytical approach is described in Supplementary Fig. 1.

\section{Pharmacological manipulation of AR signaling induces alternative splicing in prostate cancer cells}

To study the effect of pharmacological manipulation of AR signaling in prostate cancer cells, we performed expression profiling of $\mathrm{LNCaP}$ cells that were cultured for $72 \mathrm{~h}$ in charcoal-stripped fetal bovine serum and stimulated with 10-nM AR agonist, dihydrotestosterone (DHT), or $10 \mu \mathrm{M}$ of the AR-antagonist casodex for $24 \mathrm{~h}$. The array consisted of $>6$ million probes, of which $70 \%$ covered exons for coding transcripts while the remaining 30\% covered exon-exon splice junctions and noncoding transcripts; hence, allowing us to monitor transcriptional changes at the level of the exon. Besides, the well-characterized gene expression changes (Supplementary Table 1), we found that pharmacological manipulation of AR signaling induced global changes in ASE, which was evident by differential expression of exon-exon splice junction probes in comparison to constitutive exons (Supplementary Table 2). 
Figure 1a shows the expression of top-50 differentially expressed probes spanning exon-exon junction of a gene across different conditions. We next sought to characterize the potential ASE events using the transcription analysis console, which integrates the evidences from array probes spanning exon-exon splice junction and constitutive exons to classify the events as either cassette exon (CE), alternative 3 prime start site (A3SS), alternative 5 prime start site (A5SS), intron retention (IR), complex event, alternative last exon (ALE), mutually exclusive exon (MEE), or alternative first exon (AFE) (Fig. 1b). Because changes in expression at gene level may confound changes at exon-exon junction or intronic level, we filtered out any splicing events that occurred within gene that were also differentially expressed in summary, treatment of LNCaP cells with DHT, or casodex resulted in 671 and 2127 significant ASE events in comparison to DMSO treatment, respectively. Furthermore, in comparison to stimulation, inhibition of AR in LNCaP cells led to greater than 2827 ASE events. We found that treatment of $\mathrm{LNCaP}$ cells with antagonist or agonist did not drastically alter the distribution of CE (63\% vs. $84 \%)$, A3SS (12\% vs. 6\%), A5SS $(15.0 \%$ vs. $8.0 \%)$, ALE ( $0.2 \%$ vs. $0 \%)$, MEE (0\% vs. $0.1 \%)$, and $\operatorname{AFE}(0.1 \%$ vs. $0 \%)$. However, treatment with casodex did show a tenfold increase $(11.0 \%$ vs. $1.0 \%)$ in the percentage of IR events in comparison to agonisttreated LNCaP cells.

We next sought to validate the ASE events predicted by the splicing array using the RT-PCR assay. The CE and IR are some of the most common splicing events contributing toward transcriptional heterogeneity in tumor cells and are also well-characterized [36-38, 33]. Therefore, we decided to validate a total of 15 of these events in three separate cell lines including LNCaP, 22RV1 (castrate-resistant prostate adenocarcinoma cells), and PC3 (bone metastatic prostate cancer cells). We performed three independent experiments each with three technical replicates. The ASE events for validation were picked based on the following three criteria: (1) events with FDR cutoff of 0.05 and splicing index (SI) of $\geq|2|$; (2) events with evidence from not only the probes mapping to the alternatively spliced region but also those that mapped to junction surrounding it; and (3) genes with a known biological role in cancer. We used two primer pairs, one for monitoring the expression of constitutive exons within all the isoforms of a gene and another for measuring changes in the alternatively spliced region (Supplementary Table 3). The primers specific to the constitutive exons revealed that $\mathrm{LNCaP}$ cells express 15 genes, 22RV1 express 13 , and PC 3 cells express 14 of 15 genes tested. The RTPCR assay validated $47 \%, 53 \%$, and $28 \%$ of HTA-2.0 predicted splicing events in LNCaP, 22RV1, and PC3 cells, respectively (Fig. 1c, Supplementary Figs. 2-4, and Supplementary Table 4).
We next leveraged publicly available and in-housegenerated whole transcriptomic data to investigate whether treatment with enzalutamide, a more potent AR antagonist than casodex would also induce ASE in LNCaP cells. Briefly, we performed strand-specific 150-bp paired-end RNA-seq for LNCaP cells treated with vehicle or 10-nM DHT. In addition, we downloaded a dataset for enzalutamide or vehicle-treated LNCaP cells from GSE110903. Altogether, we compiled data consisting of two biological replicates per sample with $45-80$ million mapped reads per replicate. We used the rMATS computational pipeline with default settings to identify the splicing changes. The analysis revealed that DHT and enzalutamide treatment induced 198 and 167 significant ASE events, respectively, at a stringent filter of FDR $\leq 0.05$ and delta PSI of $\geq 10 \%$. The largest difference in ASE ( 5663) was observed when we compared the transcriptome of $\mathrm{LNCaP}$ treated with enzalutamide with that of DHT (Fig. 1d). Furthermore, rMATS classification of enzalutamide or DHT-induced ASE revealed that the majority of splicing events were either MEE or CE. The heatmap showing the top 100 significantly different PSI for MEE and CE across LNCaP cells treated with enzalutamide or DHT is shown in Supplementary Fig. 5A. Moreover, splicing analysis using whole transcriptome and splicing array data are reported to produce discordant results and consistent with that observation we too found a very small overlap (377/4265) between genes identified to undergo ASE upon treatment with casodex or enzalutamide (Fig. 1e). Overall, evidence from analysis of splice array, RT-PCR and whole transcriptome data support our novel observation that modulation of AR signaling alters transcriptome of prostate cancer cells by regulating ASE.

\section{Functional analysis of genes regulated at the level of alternative splicing and transcription in prostate cancer cells}

In order to study the significance of ASE in prostate cancer cells that are driven by pharmacological manipulation of AR signaling, we first queried whether treatment with enzalutamide or casodex altered splicing of prostate-cancerrelevant genes. We curated a list of 100 genes (Supplementary Table 5) that are associated with prostate cancer progression and development. The HTA-2.0 analysis showed 49 out of 100 prostate-cancer-relevant genes that showed significantly $(p \leq 0.05)$ altered splicing in response to casodex treatment of $\mathrm{LNCaP}$ cells. In addition, the rMATS analysis showed that enzalutamide significantly ( $p \leq 0.05$ ) induced splicing in 19 out of 100 prostate-cancerrelevant genes. The heatmap comparing expression of differentially expressed HTA probes spanning exon-exon 
A

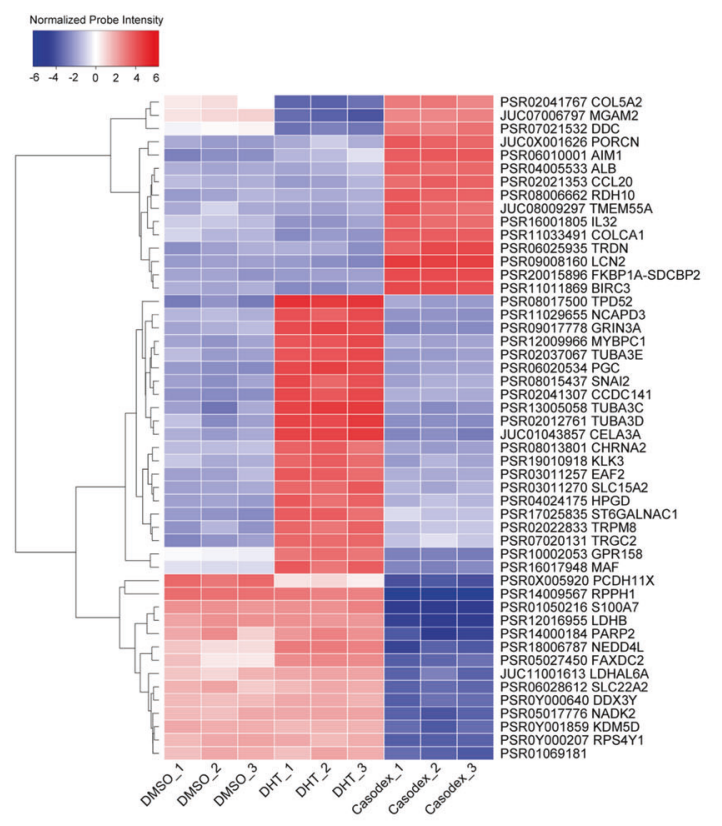

B

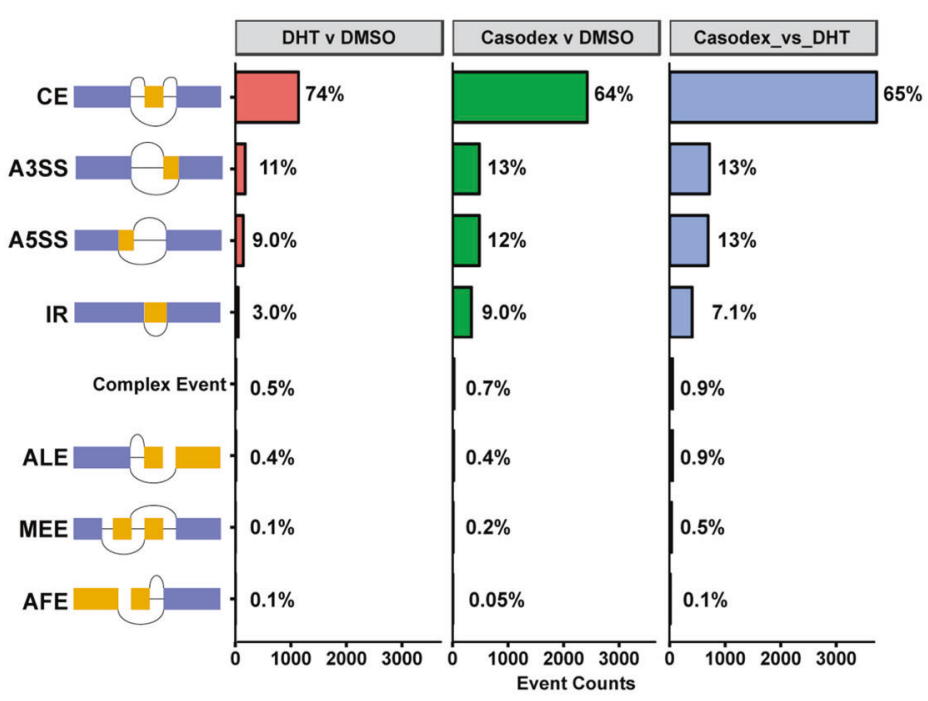

C

\begin{tabular}{llll}
\hline RT-PCR Validation & LNCaP & 22RV1 & PC3 \\
\hline No. of Genes Tested & 15 & 15 & 15 \\
No. of Genes Expressed & 15 & 13 & 14 \\
No. of Genes Validated & 7 & 7 & 4 \\
Validation Percentage & $47 \%$ & $53 \%$ & $28 \%$ \\
\hline
\end{tabular}

D

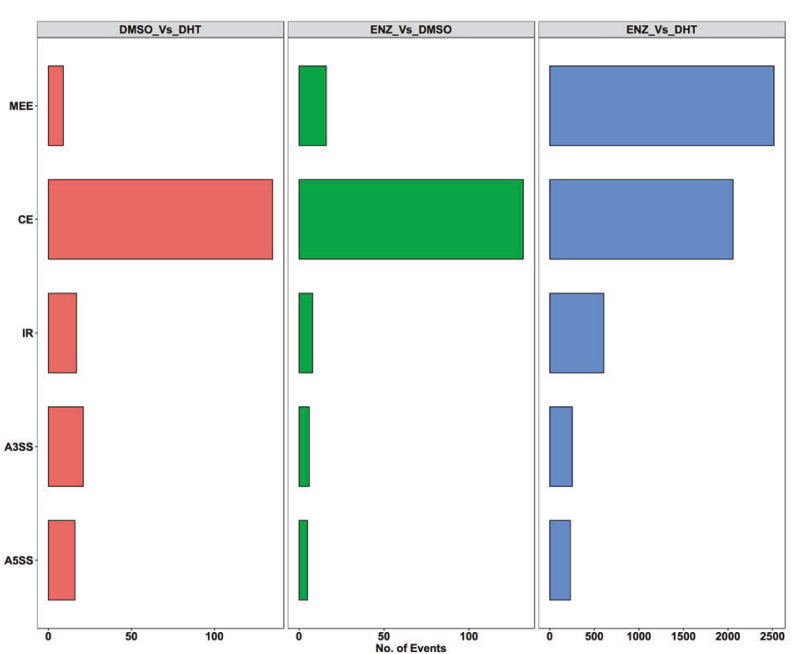

E

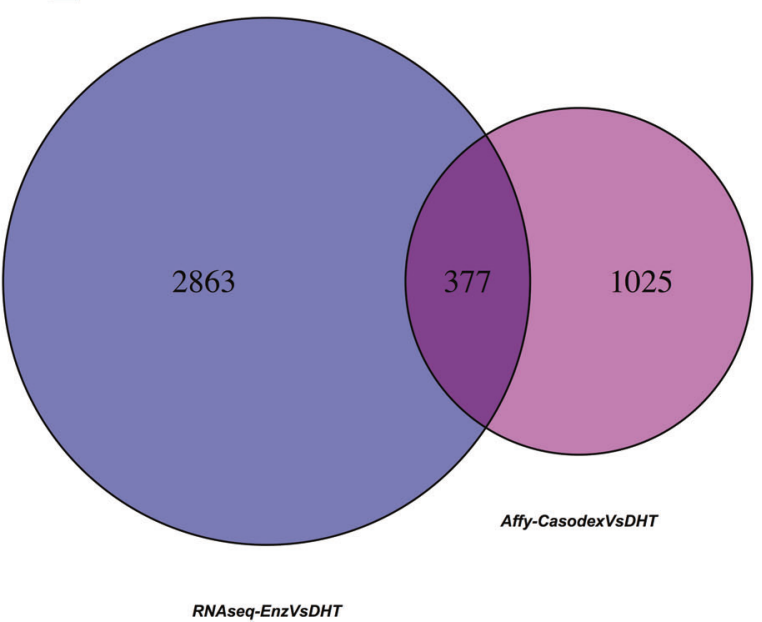

junction of a prostate cancer genes and PSI of the prostatecancer-relevant genes across samples is shown in Fig. 2a. Interestingly, a majority of alternatively spliced prostatecancer-relevant genes included key members of PI3K-AKT-mTOR pathways, which included $A K T 2$, AKT3, GSK3 $\beta$, PIK3R, PIK3CD, RB1, EGFR, PDGF,
MAPK3, KRAS, IDHI, and MTOR. IDHI was one of the RT-PCR-validated gene and is a key metabolic gene regulating TET2-mediated epigenetic re-programing in prostate tumor cells $[39,40]$. We found that casodex treatment of all three prostate cancer cell lines resulted in a switch from ENST00000345146, a dominant transcript of IDH1 to 
Fig. 1 Pharmacological inhibition of androgen receptor signaling induces alternative splicing in prostate cancer cells. a Heatmap showing the normalized probe intensity of top-50 differentially expressed probes spanning exon-exon junction of a gene across different conditions including LNCaP cells cultured for 3 days in CSFBS and treated with either vehicle (DMSO), 10-nM DHT, or 10- $\mu \mathrm{M}$ casodex. b Bar plot detailing percentage of alternative-splicing events including CE, A3SS, A5SS, IR, ALE, MEE, AFE, and complex events in LNCaP cells across three comparisons including 10-nM DHT vs. DMSO, $10-\mu \mathrm{M}$ casodex vs. DMSO, and $10-\mu \mathrm{M}$ casodex vs. 10-nM DHT. c We leveraged RT-PCR and validated 15 splicing events, which were rationally selected from the affy transcriptomic analysis in three prostate cancer cell line models. Briefly LNCaP, 22RV1, and PC3 cells were cultured in charcoal-stripped fetal bovine serum for 3 days and treated with either DMSO, 10-nM DHT, or 10- $\mu$ M casodex. The table details no. of genes tested, no. of genes expressed in each cell line, and no. of genes that were validated using the RT-PCR assay. d We leveraged in-house and publicly available data to interrogate whether enzalutamide treatment modulates ASE in LNCaP cells in comparison to DMSO or DHT treatment. Bar plot detailing percentage of alternative-splicing events including CE, A3SS, A5SS, MEE, and IR in LNCaP cells across three comparisons including DMSO vs. DHT (within-study comparison), enzalutamide vs. DMSO (withinstudy comparison), and enzalutamide vs. DHT (between study comparison). e Venn diagram comparing genes identified to undergo ASE in $\mathrm{LNCaP}$ cells treated with either casodex or enzalutamide.

ENST00000415913 with an alternate 5'UTR (Supplementary Fig. 3). The translational relevance of this functional switch was accentuated by our expression and survival analyses, which revealed that the expression of the primary isoform (ENST00000345146) is significantly higher $(p=$ $1.84 \mathrm{e}-43)$ in the TCGA-prostate adenocarcinoma tissue $(n=495)$ in comparison to the GTEX-normal prostate tissue $(n=100)$ and is also associated with decreased overall patient survival (Fig. 2b).

We further investigated whether genes modulated by casodex and DHT at the level of transcription and ASE have different physiological roles. We derived biological roles for this exclusive set of AR-axis modulated genes by using gene ontology (GO) overrepresentation analysis. We used all the genes that have an annotation as reference in our analysis as recommended by clusterProfiler. The alternatively spliced genes were enriched in pathways related to modulation of gene expression including nucleic acid, protein transport, mRNA splicing, and proteosomal degradation whereas the differentially expressed genes were enriched in pathway related to EMT including those involved in GTPase activity, cell-cell interaction/junctions, and cytoskeleton organization (Fig. 2c). We next leveraged RNA-seq data from enzalutamide and DHT-treated cells to investigate the potential mechanisms of action through which ASE may alter function of a gene. Briefly, we mapped the region undergoing ASE to protein domains of the gene using Maser $\mathrm{R}$ package. We found that the majority of splicing occurred in the characterized functional domains including the UniProt structural domains (domain), transmembrane domain, coiled region, topo domain, metal binding, zinc finger binding, and activation site for protein (Supplementary Fig. 5B). Furthermore, similar to casodex, enzalutamide treatment of LNCaP cells also dysregulated splicing of genes enriched in pathways involved in the modulation of gene expression including splicing, transport of nucleic acid, proteasomal degradation, and protein localization (Fig. 2d). Collectively, our results strongly suggest that the changes in androgen-driven ASE are biologically meaningful and distinct from the functional impact of androgen-driven gene expression changes.

\section{Direct genomic inhibition of AR in prostate cancer cells induces alternative splicing}

Our data provide evidence for the AR agonist- and antagonist-mediated regulation of ASE of pre-mRNA. However, it is possible that the observed ASE is a nonspecific effect from the pharmacological treatment of cells and not a direct effect by modulation of AR. To test this possibility, we modulated expression of AR in 22RV1 cells using siRNA and used RT-PCR assays to study altered splicing of genes including AAK1, SYNE4, and MANIA1, which were predicted to undergo ASE in response to casodex treatment. We found a robust fivefold decrease in the expression of AR in 22RV1 cells transfected with siRNA in comparison to a scrambled siRNA (Fig. 3a). Supporting our hypothesis, we found that the fivefold inhibition of $\mathrm{AR}$ altered splicing of $A A K 1$ (SI: -2.52), SYNE4 (SI: -1.36), and MANIAI (SI: -3.22) in the same direction as that of casodex (Fig. $3 \mathrm{~b}$ and Supplementary Table 4). This data support our hypothesis that ASE events induced by agonists or antagonists of AR are driven in part by direct modulation of AR.

We next leveraged publicly available (GSE110903) RNA-seq data to further study the effects of genomic inhibition of AR on ASE in MDA-PCa-2b cells, a model for advanced prostate cancer (advPC) bone metastasis cells that express PSA, AR, and are androgen sensitive. The rMATS analysis revealed that siRNA knockdown of AR in MDAPCa-2b cells induced 3841 ASE events after a stringent filtration of $\mathrm{FDR} \leq 0.05$ and delta PSI of $\geq 10 \%$. Also, similar to our observations with enzalutamide-treated LNCaP cells, we found that the MEE formed the largest fraction of ASE events induced by the siRNA knockdown in MDA-PCa- $2 b$ and were followed by CE and IR events (Fig. 3c). In addition, we used the upset plot for identifying the overlap between genes that are regulated at the level of splicing by the pharmacological inhibitors of AR in LNCaP cells or genomic inhibition of AR in MDA-PCa- $2 b$. We found an overlap of 984 genes between enzalutamidetreated LNCaP and siRNA-treated MDA-PCa- $2 b$ cells, 424 
A
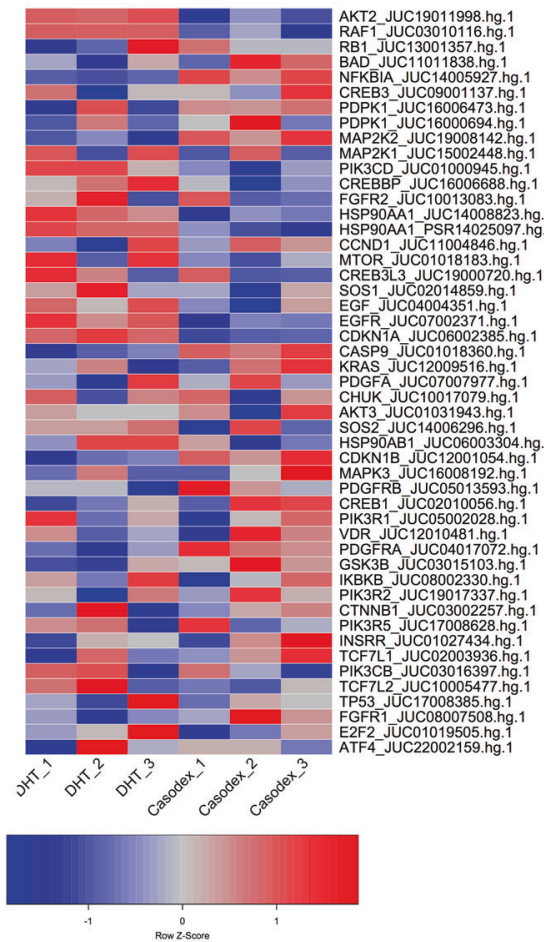

C

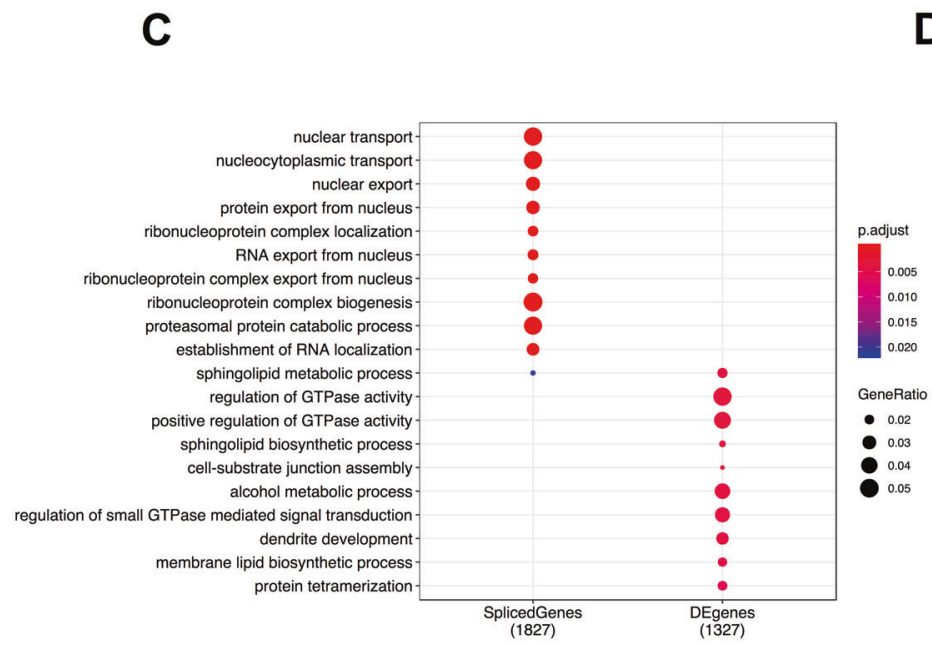

B

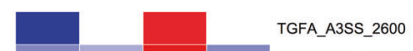
HSP90AB1_A3SS_3542 HRAS_A3SS_6184 EAF2_CE_24942 AKT1_CE_32937 AKT1_CE_32939 AKT1_CE_32940 CREB3L4_CE_59388 EGF_CE_59931 TCF7L2_CE_65579 RELA_RI_2215 NFKBIA_RI_3322 KLK3_RI_3974 GSK3B_MEE_1726 RELA_MEE_5158 PIK3CB_MEE_6071 MAP2K1_MEE_7230 MAP2K1_MEE_7232 TMPRSS2_MEE_7429 TMPRSS2_MEE_7430 TCF7L2_MEE_10694 ACSL3_MEE_12018 MDM2_MEE_13357

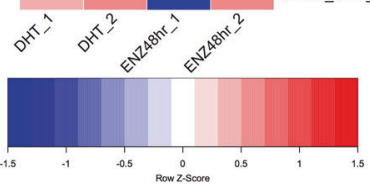

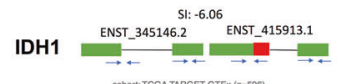

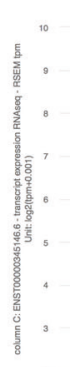

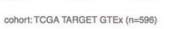
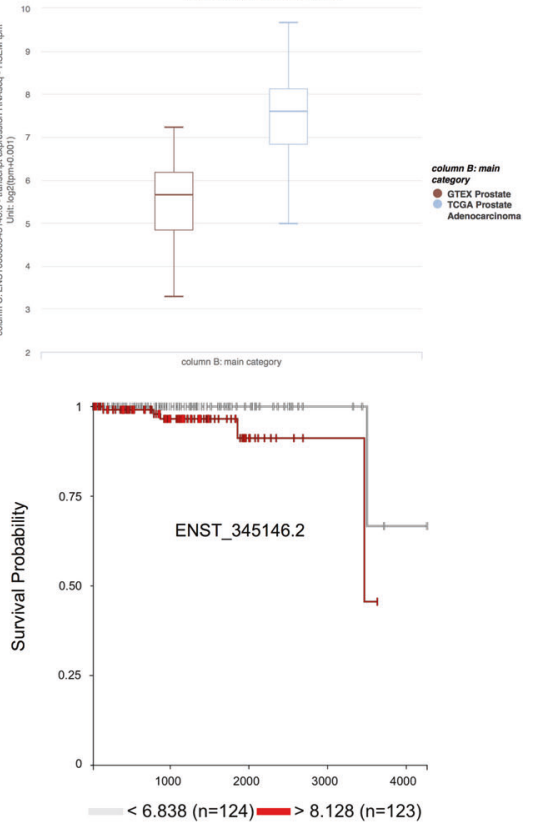

Fig. 2 Functional analysis of genes regulated at the level of alternative splicing and transcription in prostate cancer cells. a Heatmap showing the differential percent splice index (PSI) of prostatecancer-relevant genes between LNCaP cells cultured with $10-\mu \mathrm{M}$ casodex and 10-nM DHT. The adjacent heatmap shows the PSI across different conditions including LNCaP cells treated with DHT or enzalutamide. b Functional validation for the casodex-mediated switch in the IDH1 isoform expression. The box plot comparing the

genes between casodex and enzalutamide-treated LNCaP cells, and 207 genes between casodex-treated LNCaP and siRNA-treated MDA-PCa-2b cells. Interestingly, 323 genes
D

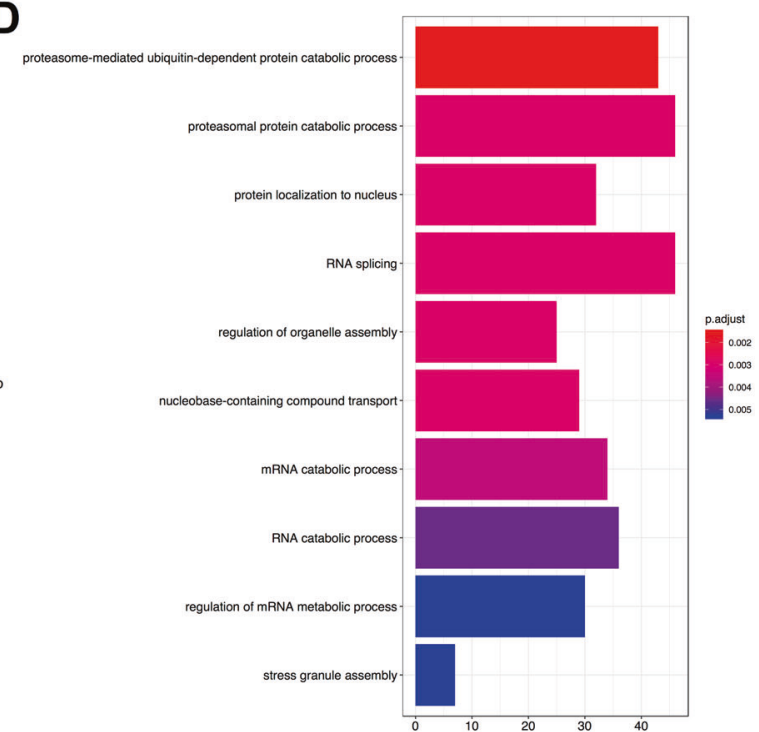

expression of the ENST00000345146 or ENST00000415913 between TCGA-prostate adenocarcinoma tissue and GTEX-normal prostate tissue. The Kaplan-Meier plot displays the association between expression of ENST00000345146 and survival for patients with prostate cancer. c A dot plot comparing GO pathways enriched in differentially expressed or alternatively spliced genes modulated by casodex and DHT. d A bar plot showing GO pathways enriched in genes modulated by enzalutamide in comparison to DHT.

were common between all three treatment groups and were enriched for pathways involved in the regulation of translation (Fig. 3d and Supplementary Fig. 5C). The plot also 
A

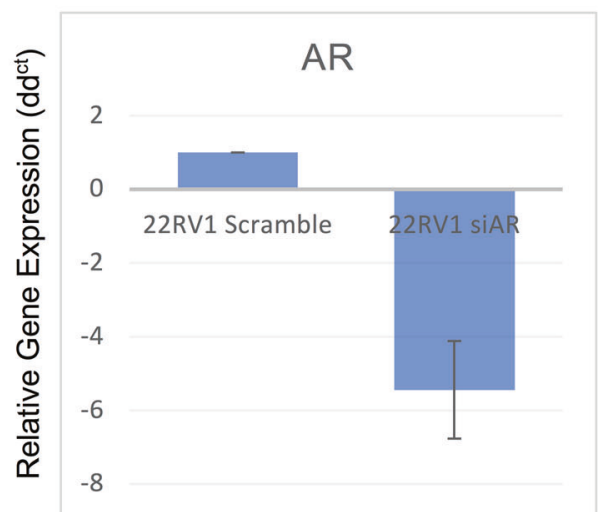

C

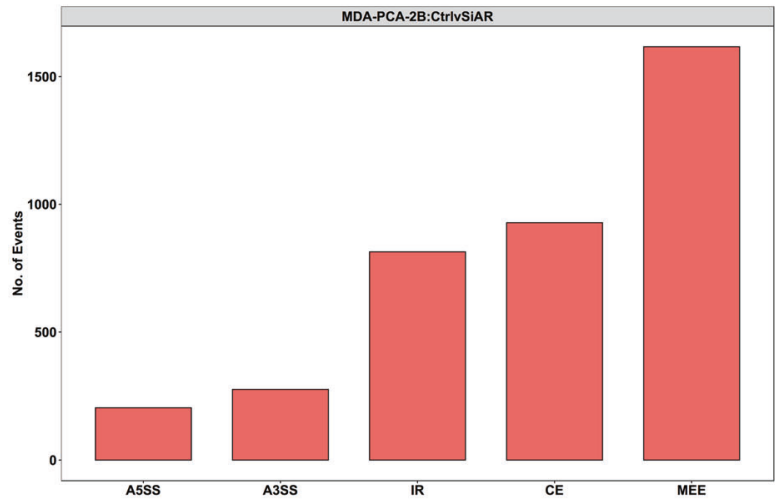

B

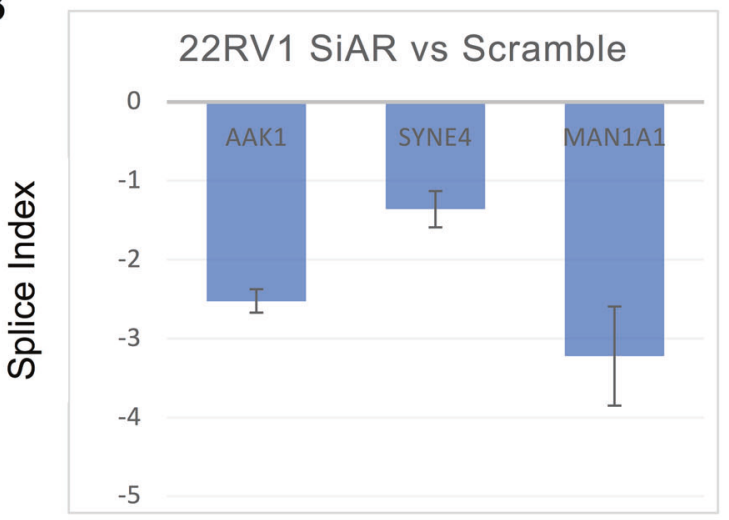

D

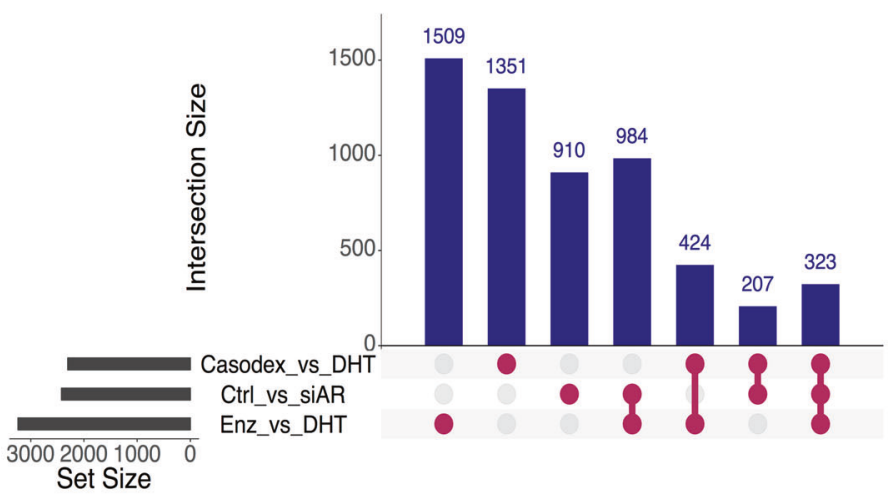

E

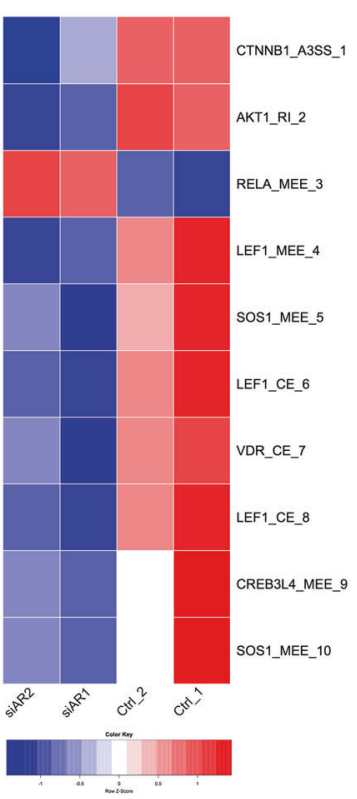

revealed a nonoverlapping set of genes in all treatment groups, possibly indicating a combination of differences in prostate cell line models and assays used for measuring ASE. The ASE genes also included known prostate-cancerrelevant genes including CTNNB1, AKT1, LEF1, and VDR.

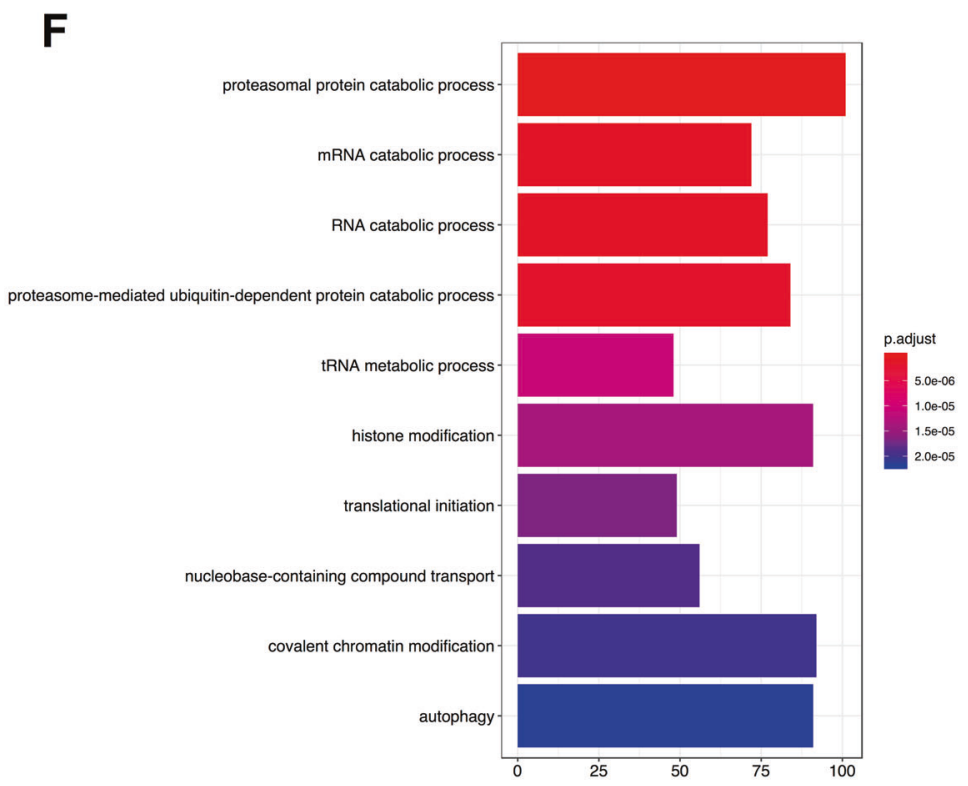

The heatmap comparing PSI for prostate cancer genes across MDA-PCa-2b cells treated with scrambled or siRNA against AR (Fig. 3e). Similar to enzalutamide-treated LNCaP cells, the GO pathway analyses revealed that genes undergoing ASE modulated by genomic inhibition of 
Fig. 3 Direct genomic inhibition of androgen receptor in prostate cancer cells induces alternative splicing. Bar graph comparing expression of AR in 22RV1 treated with scramble siRNA or siRNA against AR. b Bar graph showing expression of AAK1, SYNE4, and MAN1A1 in LNCaP cells treated with siRNA against AR in comparison to scramble siRNA. c Bar graph showing total number of rMATS predicted splicing events in MDA-PCa- $2 b$ cells treated with siRNA against AR or scrambled control. d Upset plot comparing the genes predicted to undergo alternative splicing by rMATS or HTA-2.0 analysis in prostate cancer cells treated with casodex or enzalutamide in comparison to DHT or siRNA against AR in comparison to scrambled siRNA. e The heatmap comparing PSI for prostate cancer genes across MDA-PCa-2b cells treated with scrambled or siRNA against AR. $\mathbf{f}$ Bar graph revealing the GO pathway enriched for genes under undergoing ASE modulated by genomic inhibition of AR in MDA-PCa-2b.

AR are enriched for pathways including mRNA splicing, translation initiation, chromatin remodeling, epigenetic regulation, and proteasomal degradation (Fig. 3f). In addition, the functional mapping of alternatively spliced exons revealed that genomic inhibition of AR may dysregulate function of the alternatively spliced genes by modifying functional domains (Supplementary Fig. 5D). Overall, we provide two lines of evidences supporting our hypothesis that manipulation of AR-axis either by pharmacological inhibitors of AR or by siRNA alters the splicing of premRNA, modifies the functional domain of gene, and consequentially dysregulates its function.

\section{AR-axis changes splicing of pre-mRNA by modulating expression of ESRP1/ESRP2, the master regulator of alternative splicing}

RNA binding proteins (RBPs) are key proteins that bind to mRNA or noncoding RNAs and play a wide variety of roles in posttranscriptional processing including regulation of ASE. Therefore, we hypothesized that RBPs, which are differentially expressed in response to modulation of ARaxis, might provide mechanistic insight into the observed regulation of ASE. To test our hypothesis, we curated a list of 112 RBPs from the published literature [41] with a known role in splicing regulation and investigated whether their expression was differentially regulated by treatment with casodex or DHT in LNCaP cells. Interestingly, we found that out of 112 RBPs only epithelial splicing regulator proteins (ESRP1/2) were significantly downregulated (ESRP1: $\log 2 \mathrm{FC}=-1.56 ; \quad \operatorname{padj}=7.00 \mathrm{e}-05 ; \quad \mathrm{ESRP} 2$ : $\log 2 \mathrm{FC}=-4.91 ; \quad \operatorname{padj}=5.46 \mathrm{e}-07) \quad$ in casodex-treated LNCaP cells in comparison to DHT-treated cells (Fig. 4a and Supplementary Table 1). We also leveraged STRING database and identified that the nine key binding proteins of ESRP1 and ESRP2 (protein-protein interaction enrichment $p$ value $=2.96 \mathrm{e}-08)$ were deregulated by treatment with casodex in comparison to DHT (Supplementary Fig. 5E).
ESRPs are reported to regulate splicing in a positiondependent manner. Binding of ESRPs in the downstream intron promotes exon inclusion, while binding within or upstream from an exon promotes exon skipping [42]. Accordingly, we confirmed the enrichment of ESRP1/2 binding sites downstream from AR-enhanced CEs and upstream from AR-excluded CEs in LNCaP cells treated with enzalutamide. Figure $4 \mathrm{~b}$ depicts enrichment of ESRP2 binding sites in the intronic region 0-125nt downstream of AR-enhanced exons and underrepresented in the same region downstream of AR-excluded exons. Interestingly, we found that there is underrepresentation of ESRP1 binding sites in the intronic region $0-125 \mathrm{nt}$ downstream of AR-enhanced exon and enrichment in the same region downstream of AR-excluded exons. Furthermore, ESRP2 binding motifs were enriched upstream of AR-excluded exons between -50 and -150 and underrepresented in the same region of AR-enhanced exons. In contrast, ESRP1 binding motif were enriched upstream of the AR-enhanced exons between 0 and -100 and underepresented in the same region of AR-excluded exons. Also, ESRP2 motifs were underrepresented in the region within the silenced and upregulated exons, ESRP1 motif were enriched in the upregulated exon and underrepresented in downregulated exons. This suggests that pharmacological manipulation of AR may alter splicing by regulating expression and binding of ESRP1/2 around spliced exons. Consistent with our findings, Munkley et al., recently published their findings suggesting that androgen stimulation induces splicing switches in ESRP2-controlled mRNA isoforms [43]. We also inquired whether AR-axis may regulate splicing by a nontranscriptional mechanism. For this we leveraged STRING database and identified that out of 112 RBPs, SART3 was predicted as a binding partner of AR with string high confidence level of $\geq 0.7$ (Supplementary Fig. 5F). Further supporting ESRP-independent mechanism of action AR-axis driven ASE, we found SART3 to be enriched downstream of AR-enhanced exons between 125 and 250 and underrepresented in the same region of ARexcluded exons (Supplementary Fig. 5G).

ESRP1/2 is involved in EMT and invasion of cancer cells [42, 44]. However, whether ESRP1/2 regulates EMT or invasion of prostate cancer cells has not been established. In order to study the significance of the AR-ESRP axis in prostate cancer, we employed an in vitro invasion assay. Consistent with published in vitro and in vivo report [45], we found that treatment with DHT and casodex increases the invasion rate of LNCAP and 22RV1 (Fig. 4c, d). Interestingly, when ESRP1/2 is silenced in prostate cancer cells (Fig. 4e, f), the DHT- or casodexmediated increase in the invasion rate is completely abolished. Furthermore, the genomic inhibition of ESRP1/ 2 in prostate cancer cells did not affect the expression of 
A

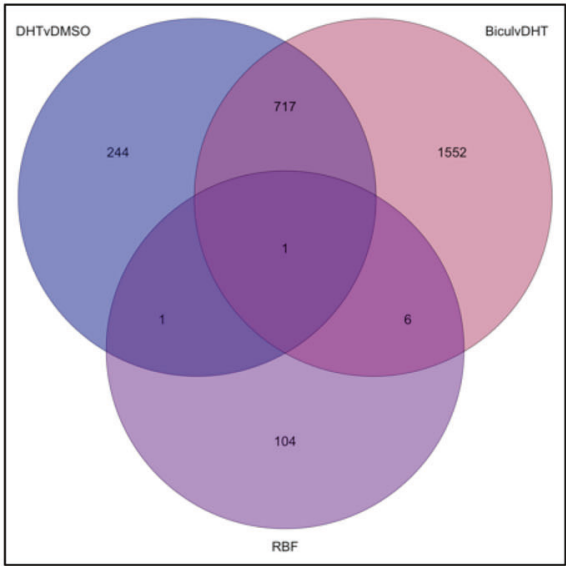

C

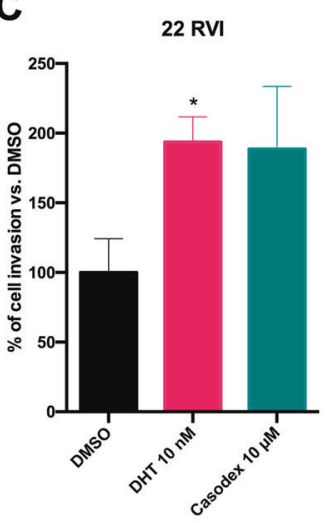

D

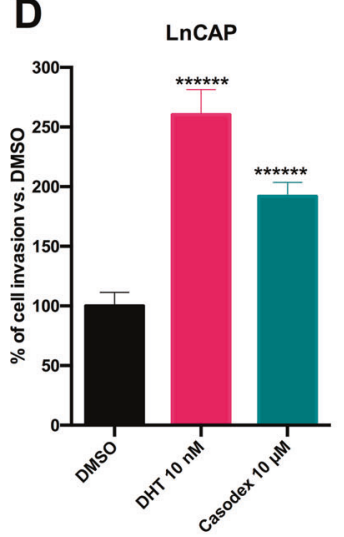

B

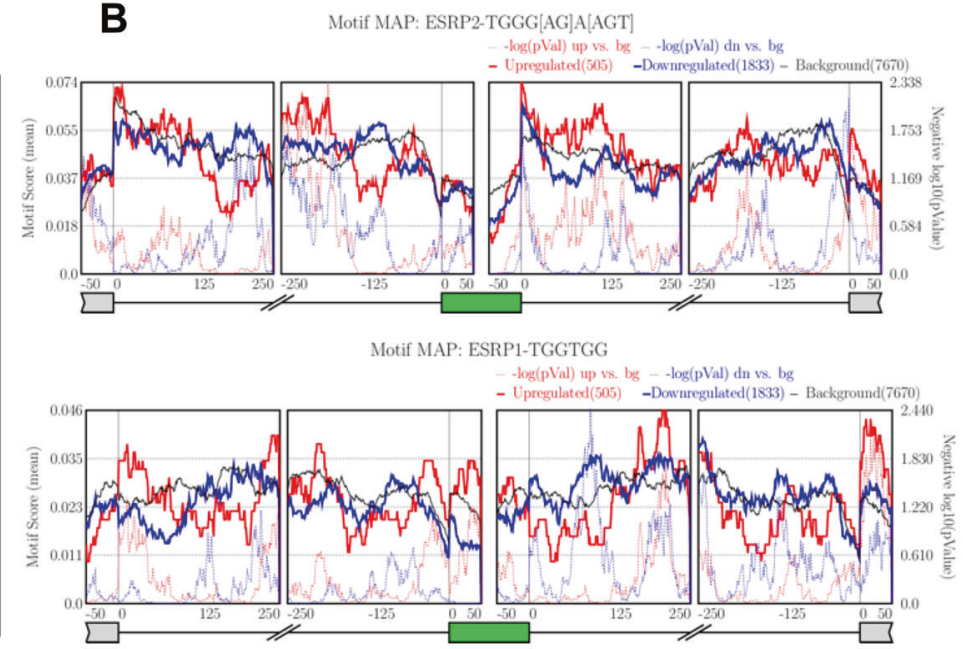

E 22 RVI (ESPR1+ESPR2 siRNA)
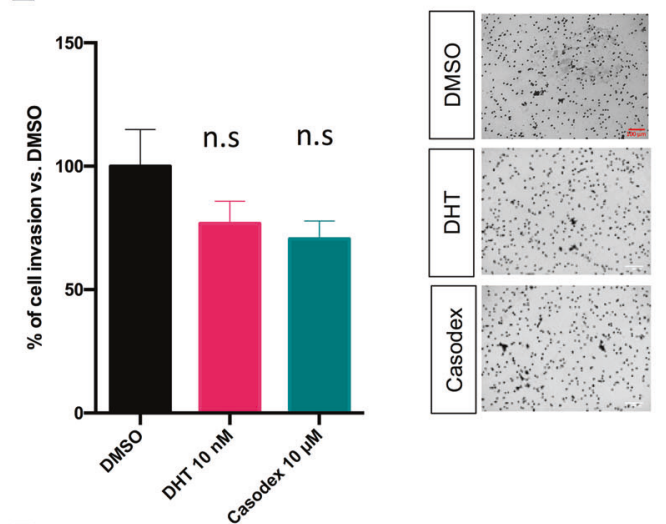

$\mathbf{F}$

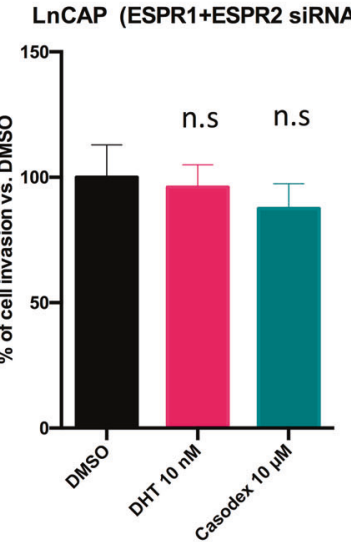

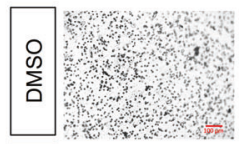

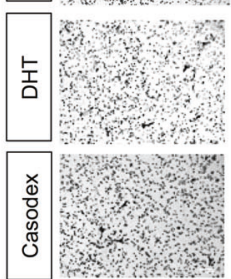

E-cadherin and vimentin, key genes involved in EMT (Supplementary Fig. 6).

\section{Modulation of AR-axis regulates splicing of pre-mRNA that are associated with progression of prostate cancer disease}

Our analysis in prostate cancer cell lines shows that modulation of AR signaling dysregulates splicing of functionally relevant genes. Since dysregulation of AR signaling is the hallmark of prostate cancer progression, we hypothesized that ASE of functionally relevant genes would be associated with the progression of disease. To test our hypothesis, we employed rMATS splicing analysis using GSE80609 [46]. This dataset consisted of 8 benign prostate hyperplasia (BPH), 16 localized prostate cancer (L.PC), 9 advPC, 12 CRPC, and 4 pairs of advPC and CRPC samples from the same patient. Since the rate for RT-PCR validation for bioinformatically predicted spliced events is low, we 
Fig. 4 Androgen receptor-axis changes splicing of pre-mRNA by modulating expression of ESRP1/ESRP2, the master regulator of alternative splicing. a Venn diagram showing an overlap between a curated list of 112 RBPs with known role in regulating alternative splicing, genes that were differentially expressed in LNCaP cells treated with DHT or casodex in comparison to DMSO. b Maps for ESRP1 and ESRP2 binding motifs showing enrichment upstream and downstream from exons upregulated or downregulated in $\mathrm{LNCaP}$ cells treated with enzalutamide in comparison to DHT. 2D invasion assay: 22 RVI $\mathbf{c}$ and LnCAP d cells were seeded on the Matrigel-coated upper chamber of the transwell insert and treated with either vehicle (DMSO) or with 10-nM DHT or 10- $\mu \mathrm{M}$ casodex in serum-free media, while $10 \%$ FBS medium was added to the lower chamber used as chemoattractant. After $24 \mathrm{~h}$, migrated cells were fixed and stained with crystal violet and counted using an inverted microscope. One-way ANOVA test was performed using Prism 8 software. Data are expressed as mean $\pm \mathrm{SEM} ; * P<0.05, * * * * * * P<0.00001$. Experiment was performed three times, with three replicates for each experiment. 22 RVI e and LnCAP f cells were transfected with indicated siRNA cell, seeded on the Matrigel-coated upper chamber of the transwell insert and treated with either vehicle (DMSO) or with 10-nM DHT or $10-\mu \mathrm{M}$ casodex in serum-free media, while $10 \%$ FBS medium was added to the lower chamber used as chemoattractant. After $24 \mathrm{~h}$, migrated cells were fixed and stained with crystal violet and counted using an inverted microscope. One-way ANOVA test was performed using Prism 8 software. Data are expressed as mean \pm SEM; no significand was detected. Experiment was performed three times, with three replicates for each experiment.

filtered our results with stringent cutoff of at least $10 \%$ difference in PSI and minimum FDR value of at least 0.05 . In concordance with Kang et al. gene-centric study, our splice analysis also found the greatest difference between the transcriptome of BPH and L.PC and lowest between that of advPC and CRPC (Fig. 5a). In particular, we found that 53 A3SS, 46 A5SS, 574 CE, 191 IR, and 277 MEE differentiated BPH from L.PC (A); 25 A3SS, 13 A5SS, 155 CE, 81 IR, and 128 MEE events differentiated L.PC from $\operatorname{advPC}(\mathrm{B}) ; 8$ A3SS, 10 A5SS, 73 CE, 20 IR, and 14 MEE differentiated advPC from CRPC (C); and 10 A3SS, 20 A5SS, $72 \mathrm{CE}, 37 \mathrm{IR}$, and 2 MEE differentiated paired samples of advPC and CRPC (D) (Fig. 5a).

The earlier study identified dysregulation of AR expression as the only shared event across all stages of prostate cancer [46]. To the contrary, we found a total of nine splice events to be associated with all stages of prostate cancer $(\mathrm{A} \cap \mathrm{B} \cap \mathrm{C} \cap \mathrm{D})$ (Supplementary Fig. 7A-C). These events included CE and MEE in the pre-mRNA of cancerrelevant genes, including TRIM37, PTGR1, CREM, HMOX2, UPF3A, TNRC6B, PLA2G2A, and ESYT2. The direction of PSI for these events varied during disease progression, suggesting a differential role for these genes in disease (Fig. 5b). Supplementary Fig. 7D-L shows the transcript plots displaying the usage of exon for these nine genes between AdvPC and CRPC. The transcriptome of advPC and CRPC is reported to be highly similar and previous study had identified only 15 genes that differentiated their transcriptome. Hence, we further investigated whether ASE events may further distinguish advPC from CRPC. We compared the list of splicing events in paired and unpaired samples of advPC and CRPC $(\mathrm{C} \cap \mathrm{D})$ and found 13 unique splicing events that differentiated the transcriptome of advPC and CRPC (Supplementary Fig. $7 \mathrm{~A}-\mathrm{C}$ ). These splicing events occurred in the pre-mRNA of PTGR1, FRGIHP, RP11, CA5BPI, CREM, TNRC6B, BCS1L, FASTKD1, ESYT2, CLN3, PLA2G2A, MYL6, FBXL12, ZNF202, UBAP2, and MIR940 (Supplementary Fig. $7 \mathrm{M}$ ). The rMATS analysis of the prostate cancer dataset identified that ASE is associated with tumorigenesis, advanced progression, and CRPC development. Furthermore, in addition to differentially expressed genes, our study identifies splicing events that can further differentiate the transcriptome of advPC and CRPC.

Our analysis shows that pharmacological or genomic inhibition of AR alters splicing of several functionally relevant genes. Therefore, it is possible that a subset of these alternatively spliced genes is associated with progression of prostate cancer. To test this possibility, we compared the list of splicing events common across all stages of prostate cancer $(\mathrm{A} \cap \mathrm{B} \cap \mathrm{C} \cap \mathrm{D})$ and list of splicing events induced by either pharmacological and genomic inhibition of AR in LNCaP and MDA-PCa-2b cells (Supplementary Fig. 7A-C). We found only one significant event that alters the inclusion of exon-2 (Chr1:19979580-19979659) in the pre-mRNA of PLA2G2A, a secreted phospholipase. PLA2G2A is significantly downregulated in patients with metastatic prostate cancer in comparison to the primary tumor. Moreover, the decrease in expression of PLA2G2A is implicated in promoting invasion and metastasis [47]. Interestingly, exon-2 of PLA2G2A contains a repressor region, and its inclusion is associated with a decrease in the expression of the gene $[48,49]$. In support of the known role of $P L A 2 G 2 A$, we found an increase in the inclusion of exon-2 in advPC in comparison to CRPC (paired and unpaired samples), BPH in comparison to L.PC (unpaired samples) and in response to the pharmacological or genomic inhibition of AR signaling in LNCaP and MDA-PCa-2b cell lines, respectively (Fig. 5c).

To study the functional significance of the exon-2 splicing, we performed expression and survival analysis with the TCGA-PRAD and GTEX datasets. In particular, we found that percentage of the ENST00000375111.7 isoform, that contains exon-2 was significantly higher in patients with prostate cancer $(n=496$, TCGA-PRAD) in comparison to healthy prostate tissue ( $n=100$, GTEX-prostate). In contrast, the percentage of the ENST00000400520.7 isoform that does not contain exon- 2 was significantly lower in patients with prostate cancer in comparison to healthy prostate (Fig. 5d). In addition, the survival analysis for primary prostate cancer patients in TCGA dataset revealed 
A

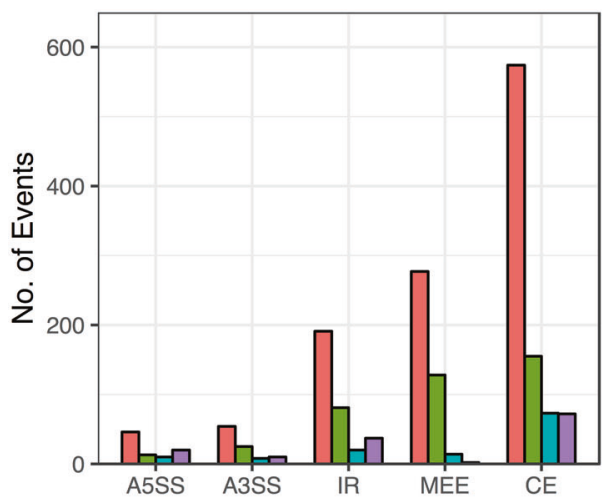

C

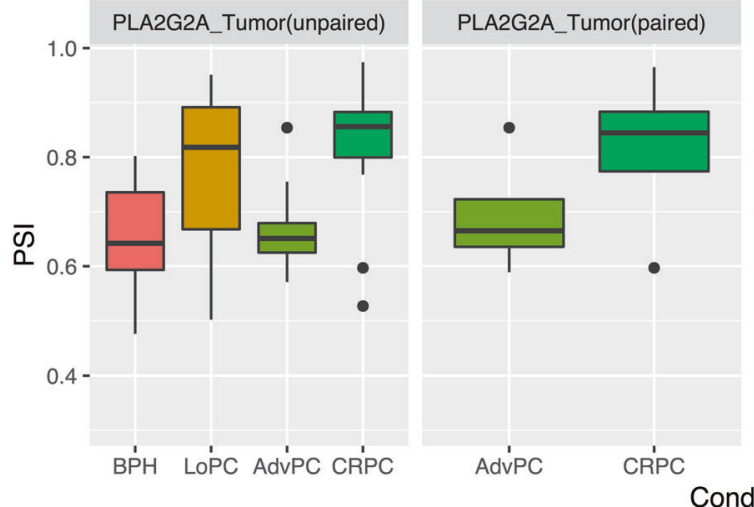

Condition
B
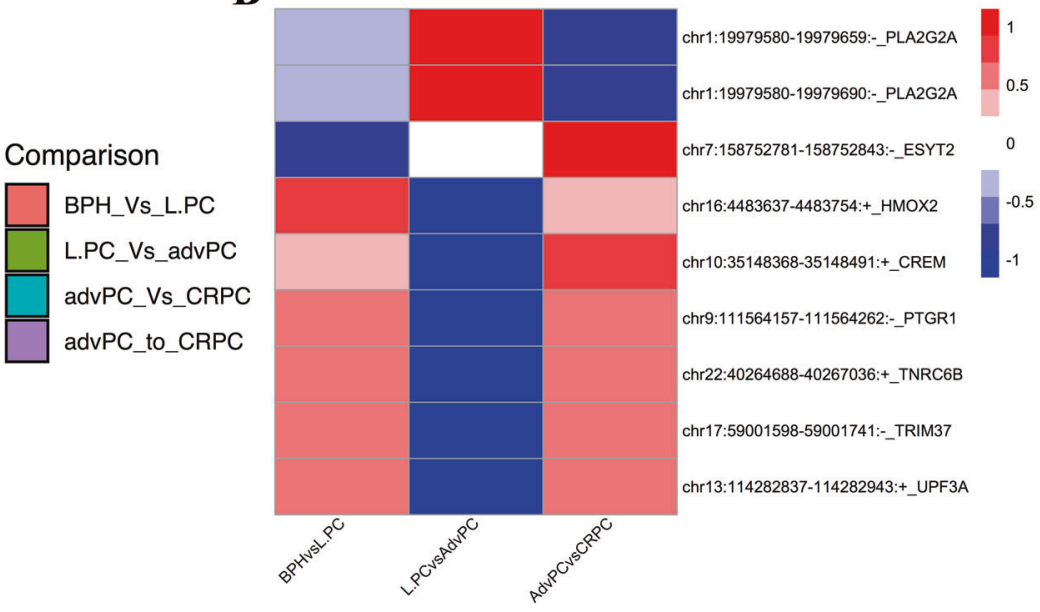

D

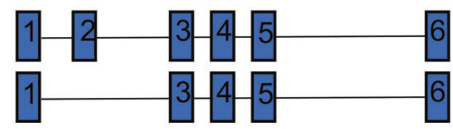

ENST_111.7

ENST_520.7

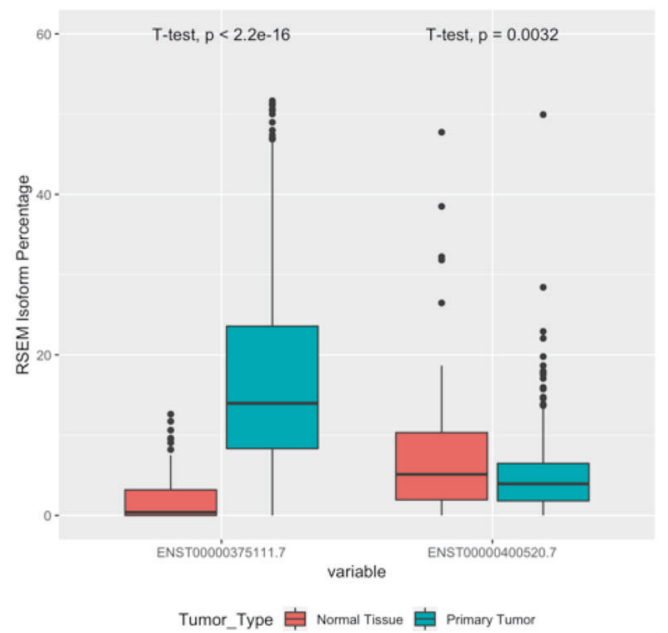

that patients with primary tumors with a higher difference between percentage isoform of ENST00000375111.7 than ENST00000400520.7 ( $>18.17 ; n=122)$ had significantly ( $p=0.021)$ shorter overall survival compared to those difference of $<4.08(n=123)$ (Fig. 5e). In conclusion, we

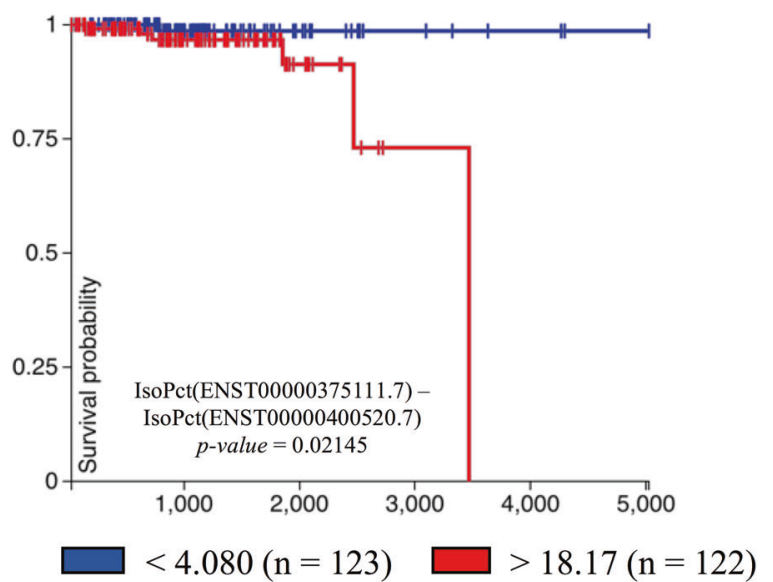

show that differential usage of exon or introns is associated with progression of prostate cancer. In addition, inhibition of AR may inadvertently switch the delicate balance between different splice isoforms in genes critical for disease progression. 
Fig. 5 Modulation of AR-axis regulates splicing of pre-mRNA that is associated with progression of prostate cancer disease. a Bar graph comparing significant number of splicing events across patients at different stages of prostate cancer including benign prostate hyperplasia (BPH), localized prostate cancer (L.PC), advanced prostate cancer ( $\operatorname{advPC})$, castrate-resistant prostate cancer (CRPC), and four pairs of advPC and CRPC samples from the same patient. b Heatmap comparing the PSI for the cassette or mutually exclusive exons across patients at different stages of prostate cancer. $\mathbf{c}$ Box plot displaying the changes in PSI of the exon-2 of PLA2G2A gene in patients at different stages of prostate cancer or in prostate cancer cells treated with DMSO, DHT, Enzalutamide, scrambled siRNA, or siRNA against AR. d The box plot comparing the expression of the ENST00000375111.7 or ENST00000400520.7 between TCGAprostate adenocarcinoma tissue and GTEX-normal prostate tissue. e The Kaplan-Meier plot displays the association between overall survival for patients with prostate cancer and difference in expression of percentage isoform of ENST00000375111.7 than ENST00000400520.7.

\section{Discussion}

In this study, we leveraged a combination of nextgeneration sequencing and molecular methodologies to provide critical pieces of evidence for a direct role of AR and its clinical inhibitors in regulating the transcriptome of prostate cancer cells by modulating global alternative splicing. A key finding from our study is that AR-driven alternatively spliced genes had a distinct physiological role from those that were transcriptionally regulated by AR in prostate cancer cells. Furthermore, we found that antiandrogens currently used in clinics may inadvertently contribute to the disease's progression by dysregulating splicing. Given that prostate cancer patients on antiandrogens will only experience 8-19 months of progression-free survival [29], our study provides one possible explanation for disease progression on these therapies.

ASE is known to play a significant role in maintaining cellular physiology. Therefore, identifying the transcriptional splicing patterns may have the potential for early diagnosis, prognosis, and identification of therapeutic targets in tumor biology [50]. The nuclear hormone receptors, including estrogen and progesterone receptors, are known to modulate ASE. However, the role of AR in modulating ASE remains mostly unexplored. Munkley et al. recently used the genomic knockdown of ESRPs to identify global splicing changes associated with ESRPs in prostate cancer cells. They also found that a subset of ESRP1/2 regulated exons were also regulated by androgen treatment [43]. However, the study did not address whether or not AR-axis induces global splicing changes in prostate cancer cells beyond those regulated by ESRPs. Therefore, there remains a gap in our understanding of AR signaling and if it can regulate the transcriptome of prostate cancer cells by altering global splicing levels. In this study we focused on whether genomic and pharmacological inhibition of $A R$ expression and AR activity, respectively, could regulate global ASE in prostate cancer cells. We also explored prognostic and functional implications of splicing induced by genomic and pharmacological manipulation of AR currently used in the clinic.

We leveraged HTA-2.0, a newer generation of microarray that interrogates junctions between exons in the transcriptome as well as the exon themselves and RNA-Seq analysis to test whether modulating AR signaling would alter ASE prostate cancer cells. This approach leds to an unexpected finding that treatment with DHT or casodex induces a large number of ASE in prostate cancer cells. We confirmed our observations by conducting a thorough validation of predicted splice events using real-time PCR in three different prostate cancer cell lines, including androgen-sensitive LNCaP, castrate-resistant 22RV1, and metastatic PC3 cell lines. We also leveraged in-house and publicly available RNA-Seq data to validate whether inhibiting AR signaling with enzalutamide would also induce ASE. Indeed, our analysis found that enzalutamide in comparison to DMSO control induced ASE in LNCaP cells. Similar to casodex experiment, the highest number of ASE was observed when a comparison was made between enzalutamide- and DHT-treated LNCaP cells. However, a comparison between DHT- and enzalutamide-treated LNCaP cells involves comparing experiments from different groups; therefore, we cannot rule out the possibility of a batch effect in this comparison. Given the limitations of across study comparison, we also performed a within study comparison between LNCaP cell treated enzalutamide and DMSO. Supporting our hypothesis that treatment with AR signaling modulates alternative splicing, we found that enzalutamide induced $>150$ ASE in comparison to DMSO treated LNCaP cells. Further experiments would be needed to validate observed changes between DHT- and enzalutamide-treated prostate cancer cells. In conclusion, we leveraged in vitro experiments and publicly available genomic data to support our findings that AR signaling may alter the transcriptome of prostate cancer cells by modulating global ASE.

AR molecules in the cytoplasm dimerize and translocate to the nucleus in response to androgens. Dimerized molecule of AR binds to ARE and regulates target gene expression. Therefore, a majority of published reports have primarily focused on genes transcriptionally regulated by AR to understand the physiological significance of AR signaling. Consequently, we tested whether the ARsignaling-regulated ASE has a different physiological role from differentially expressed genes. Consistent with earlier findings, we found that inhibiting AR signaling leds to expression changes of genes involved in EMT [51, 52]. However, contrary to the report from Munkley et al., which proposed that AR may regulate splicing of essential EMT 
related genes, we did not find AR-axis-driven ASE enriched for EMT-associated pathways. Instead, we discovered that AR-axis-driven ASE were enriched in pathways involving gene expression regulation, nucleic acid and protein transport, mRNA splicing, and proteasomal degradation. The differences observed could be driven by the fact that while previous work focused on the genomic knockdown of ESRPs, our work exclusively focused on modulating AR expression or its activity to identify AR-axis-driven ASE events. Because we filtered out alternatively spliced genes, which were also differentially expressed, we were able to discover a new and unique physiological impact of modulating AR signaling in prostate cancer cells. The role of AR-regulated splicing is further signified by our analysis showing that the majority of splicing occurred in characterized functional domains, including the transmembrane domain, coiled region, topo domain, metal binding, zinc finger binding, and activation sites. The lack of overlapping functional role between differentially expressed and ASE could be explained by the fact that we had excluded ASE occurring in genes that were also regulated transcriptionally.

Interestingly, we observed that inhibiting AR signaling using casodex and enzalutamide significantly increased IR compared to DHT treatment. IR is known to generate abnormal transcripts that are translated into immunogenic peptides, loaded on MHC-1, and presented to the immune system [53]. Therefore, patients with advanced-stage prostate cancer undergoing treatment with AR inhibitors may have a higher neoepitope load and hence benefit from immune checkpoint inhibitors. Further studies will be necessary to predict and validate the immunogenicity of neoepitopes generated in response to AR inhibitors, including identification of $\mathrm{T}$ cells infiltrating prostate tumors specific to predicted neoepitopes.

The results from our work suggest that AR agonists and antagonists may dysregulate ASE within the pre-mRNA of genes that regulate tumor biology. Because pharmacological modulators may have nonspecific activity, it is possible that the observed ASE observed in response to treatment with AR modulators is not mediated through AR. Therefore, using RT-PCR we demonstrated that genomic inhibition and pharmacological inhibition of AR may induce similar splicing pattern, within a subset of genes in prostate cancer cells. In addition, we also found a significant overlap as well as unique ASE events induced in different prostate cancer cells treated by pharmacological inhibitor or the genomic inhibition of AR. For our global splicing analysis, we leveraged multiple prostate cancer cell lines; therefore, some of the common or unique ASE events identified could be because of cell line differences and not an effect of treatment. Therefore, the global comparison of ASE between the pharmacological inhibitor and genomic inhibition of AR signaling in prostate cancer cell lines needs further validation. Also, we may not be able to rule out the possibility of nonspecific pathways being engaged by pharmacological inhibitors of AR to dysregulate splicing. However, based on analytical models that predict splicing in response to genomic and pharmacological inhibition and RT-PCR validation provides sufficient evidence to conclude that ASE events induced in response to treatment with AR inhibitor are in parts driven by direct modulation of $\mathrm{AR}$ expression.

The regulation of ASE is primarily mediated by RBPs that interact with sequences flanking exon and introns as splicing enhancers or silencers, depending on the regulator and binding context. Because AR is a transcription factor, we argued that AR might regulate ASE by transcriptionally regulating the expression of RBPs. We tested 112 wellcharacterized RBPs and found that expression of ESRP1/2 in prostate cancer cells decreased in response to treatment by pharmacological inhibitors of AR. Interestingly, expression of proteins predicted to bind with ESRP1/2 was also deregulated by inhibition of AR signaling. We further confirmed that ESRP1/2 binding sites were enriched downstream from AR-enhanced CEs and upstream from AR-excluded CEs in response to pharmacological inhibition of AR in LNCaP cells. In support of our findings, Munkley et al. also reported that AR controls expression of ESRPs to regulate ASE in prostate cancer cells. However, contrary to the model proposed, we show that the AR-ESRP axis may be only of the mechanisms by which AR may regulate splicing events. To that extent, we found that the SART3, a RNA binding protein and a binding partner of AR, was predicted to be enriched downstream of AR-enhanced exons and underrepresented in the same region of ARexcluded exons in prostate cancer cells. The prediction of AR-SART3 axis being involved in regulating subset of ASE in prostate cancer cells suggests that AR may employ multiple mechanisms to modulate global splicing and they need to be carefully investigated. We will need to perform ChIP-Seq to directly validate whether androgens or antiandrogens dysregulate ESRP and SART3 binding around AR-axis driven CEs. Also, it would be interesting to study whether AR and ESRP or SART3 bind within the same region and are part of a protein complex regulating splicing.

Because ESRP plays a critical role in EMT and tumor invasion in several tumor types [44], we hypothesized that the AR-ESRP axis might be critical for promoting metastasis either by promoting EMT or promoting invasion. Moreover, although pharmacological inhibitors of ARsignaling suppress tumor growth, we and others have shown using in vitro and in vivo approaches that it also promotes invasion of tumor cells [45]. Hence, it is critical to identify new therapeutic targets that may alleviate accidental invasion promoting effects of inhibiting AR signaling in advanced state prostate cancer. Our in vitro invasion assay 
found that silencing ESRP protein abrogates casodexmediated increase in the invasion of prostate cancer cell lines. ESRPs are reported to be involved in EMT. Therefore, we analyzed E-cadherin and Vimentin, which are among the most prominent markers involved in EMT and have found no detectable differences in their expression in response to treatment with siRNA against ESRP1/2. This is contrary to the model proposed by Munkley et al., which hypothesized that downregulation of ESRP2 may decrease epithelial splicing pattern and prime prostate cancer cells for future development of mesenchymal phenotype. However, Munkley et al. did not assess whether genomic knockdown of ESRP1/2 resulted in changes in EMT marker genes (Ecadherin or Vimentin) in prostate cancer cells. Conversely, they did not find any significant association between increase in expression of ESRP1/2 in prostate carcinoma and E-cadherin levels. It is possible that more evident sign of the activation of EMT may be visible in our assay after a longer period of incubation as reported by previously published evidence using the same cell lines in which EMT was analyzed after 48 or $72 \mathrm{~h}[54,55]$. However, to be consistent with the invasion assay time frame, we analyzed cells $24 \mathrm{~h}$ after treatment. Based on findings from our study, ESRPs as a therapeutic target in combination with AR inhibitors may warrant further investigation as it provides a potential approach to counter the invasive phenotype accidentally promoted by treatment with AR inhibitors.

Our work using prostate cancer cell lines showed that AR signaling dysregulates the splicing of functionally relevant genes. Because AR signaling is a hallmark of prostate cancer progression, we also investigated whether AR-regulated ASE is associated with the progression of disease. Our analysis showed that significant splicing events are occurring during different stages of prostate cancer progression. Contrary to an earlier study [46], which had found that dysregulation of the expression of AR was the only event associated with all stages of prostate cancer, we found an additional nine splicing events. Therefore, our work also reveals a new potential area of inquiry into the underlying biology of prostate cancer initiation and progression to the castrate-resistant stage.

Furthermore, the transcriptomic analysis had only identified 15 genes that were differentially expressed between advPC and CRPC, thus making these two stages of prostate cancer challenging to differentiate. By focusing on ASE, we have now identified an additional 13 genes that are differentially spliced between advPC and CRPC. Thus, a combined gene expression and splicing panel could potentially have a higher diagnostic value to discriminate patients in an advanced stage from the one with the castrate-resistant disease.

Lastly, we investigated whether the treatment of prostate cancer cells with pharmacological inhibitor enzalutamide may lead to an inadvertent switch in the splicing of a pre-mRNA and promote tumor progression. Our analysis found that dysregulation of AR signaling in prostate cancer driven by enzalutamide treatment increases the inclusion of exon-2 of the PLA2G2A gene in prostate cancer cells. The translational significance of the inclusion of exon- 2 was validated in the TCGA-PRAD, where we revealed that the PLA2G2A isoform that includes exon-2 is a prognostic factor for outcomes, providing strong evidence for developing a therapeutic strategy that can mitigate the inadvertent pro-tumorigenic effects of inhibiting AR signaling.

In conclusion, this study highlights the so-far undescribed role of AR in modulating gene expression via ASE in prostate cancer. This discovery also opens a new therapeutic path and supports the rationale for using ESRP modulators in combination with $\mathrm{AR}$ antagonists for the treatment of advanced-stage prostate cancer to counteract the AR-antagonist-driven invasive phenotype.

\section{Materials and methods}

\section{Reagents}

$5 \alpha$-DHT, casodex, and Enzalutamide were obtained from Sigma and were resuspended in DMSO (Sigma). Primers were designed manually and were purchased from Integrated DNA Technologies. Anti-ESRP1 (\#21045-111-AP) and anti-ESRP2 (\#23317-1-AP) were obtained from the Proteintech. E-Cadherin (4A2) (\#14472) and Vimentin (D21He) (\#5741) were obtained from the CellSignaling, while anti-aTubulin antibody (\#A01410) was obtained from Genscript. All other reagents if not specified were obtained from Thermo fisher Scientific.

\section{Cell culture}

The human cell lines LNCaP (androgen-sensitive human prostate adenocarcinoma cells) and 22RV1 (human prostate carcinoma epithelial cell line derived from a xenograft that was serially propagated in mice after castrationinduced regression and relapse of the parental, androgendependent CWR22 xenograft) were obtained from ATCC and were cultured in RPMI-1640 medium. PC3 (metastatic prostate cancer cells isolated from bones, ATCC) was maintained in F-12K medium. All culture medium was supplemented with $10 \%$ HyClone Defined Fetal Bovine Serum (GE Healthcare) and 1\% Pen/Strep (Invitrogen) unless specified. Cell cultures were tested every 6 months for cross-contamination using human STR profiling cell authentication service provided by ATCC. Mycoplasma contamination was tested using MycoAlert Mycoplasma Detection Kit (Lonza). 


\section{Western blotting}

Cells were dissolved in RIPA buffer (sigma). Protein concentration was measured by BCA protein assay reagent kit (Pierce, Rockford, IL, USA), as described previously. Proteins were fractionated on $10 \%$ SDS-PAGE, and transferred by electrophoresis to nitrocellulose transfer membrane (GE). Membranes were incubated with primary antibodies for overnight. Horseradish peroxidase-conjugated antibody anti-mouse $\operatorname{IgG}$ and anti-rabbit $\operatorname{IgG}$ (Dako) were used to detect immunoreactive bands and binding was revealed using enhanced chemiluminescence (Pierce). The blots were then stripped and used for further blotting for control antibody. Unless otherwise specified, displayed western blots are representative images of at least two independent experiments.

\section{siRNA transfection}

22RV1 cells were transfected with a mixture of four different siRNA targeting AR (siRNA \#L-003400-00-0010) and non-targeting control pool (\#D-001810-10-05). PC3, $\mathrm{LNCaP}$, and 22RV1 cells were transfected with a mixture of four different siRNA targeting ESRP1/ESRP2 kinases and negative control (SiGenome ESPR1 \#D-020672-01, OnTarget ESPR2 \#J-014523-05, Darmacon, and Silencer Negative control, \#4390843 Thermo fisher). Cancer cells were seeded in a six-well plate. 200-nM siRNA/well were used for transfection using $5 \mu \mathrm{l} /$ well of Hi-Perfect (Qiagen) following manufacturer's recommendation.

\section{D invasion assay}

Reduced growth factors Matrigel (BD Biosciences) was polymerized in $8-\mathrm{mm}$ pore cell inserts (Sarstedt) prior to the addition of cells. LNCaP and 22RV1 $(5 \times 106$ cells) were seeded into the insert containing Matrigel in serumfree media. Twenty percent FBS medium was used as an attractant in the bottom chamber, and cells were allowed to migrate for additional $24 \mathrm{~h}$. The inserts were removed, and migrated cells were fixed with $4 \%$ paraformaldehyde and stained with crystal violet. The inserts were then imaged, and migrated cells were counted, hence, providing a quantitative value for migrated cells across the membrane.

\section{Human transcriptomic array}

LNCaP cells were cultured for 3 days in the RPMI-1640 with $10 \%$ charcoal-stripped fetal bovine serum and treated with either 10-nM DHT, 10- $\mu$ M casodex, or DMSO for $24 \mathrm{~h}$. Total RNA was extracted using the RNeasy mini kit (Qiagen) and quantified using Nanodrop ND-100
Spectrophotometer (Thermo Fisher Scientific). The quality of RNA was assessed using Agilent 2100 Bioanalyzer (Agilent). Biotinylated cDNAs were prepared from a minimal 100 ng of total RNA using Life Technologies WTplus RNA Amplification system (Ambion). Following the amplification, cDNA was fragmented, hybridized on Affymetrix GeneChip Human Transcriptome Array 2.0 (HTA2.0) chips, and nonspecific bindings were washed as per manufacturer's recommendations. The fluorescence intensity of the arrays was scanned using the Affymetrix Scanner and the raw data. The raw data were analyzed using the Transcriptome Console Software (TAC 2.0) that allows for the identification of differentially expressed genes and leverages information from the junction and exons probes to detect ASEs and possible transcript isoforms that may exist in samples.

For microarray data analysis, two parallel analysis (gene-level and alternative-splicing level) were performed using HTA-2.0. Data were normalized using quantile normalization and the background was detected using the built-in detection above background algorithm (DABG). Only the probesets that were characterized by a DABG $p$ value $<0.05$ in at least $50 \%$ of the samples were considered as statistically significant. Principal component analysis was used to calculate variance and confirm if the variance is similar between the groups being compared. Genes were considered to be differentially expressed when fold change (FC), $\log \leq-2.0$ or $\geq+2.0$ and FDR corrected $p$ value $\leq 0.05$. The splicing level analysis as also carried out using TAC 2.0 software, which determines the SI of a gene. The SI corresponds to a comparison of gene-normalized exon-intensity values between the two analyzed experimental conditions. Additional criteria used besides SI: FDR corrected $p$ value $\leq 0.05$, a gene is expressed in both conditions tested, a probeset ration (PSR)/junction must be expressed in at least one condition, a gene must contain at least one PSR value, and a gene cannot be differentially expressed.

\section{Reverse transcription PCR validation for splicing events}

A total validation of 15 splicing events was performed on three prostate cancer cell lines including LNCaP, 22RV1, and PC3 at various disease state (primary prostate cancer, castrate-resistant, and metastatic prostate cancer). Briefly, the prostate cancer cells were cultured for 3 days in $10 \%$ CSFBS and were treated with either DMSO, 10-nM DHT, or $10-\mu \mathrm{M}$ casodex for $24 \mathrm{~h}$. Total RNA was extracted, and cDNA was prepared using SuperScript IV First-Strand Synthesis System (Thermo Fisher Scientific). cDNA samples were amplified using SYBR ${ }^{\circ}$ Green PCR Master Mix on the Applied Biosystems 7500 Detection System. 
Spicing-specific primers included two primer pairs, one for monitoring the expression of constitutive exons within all the isoforms of a gene and another for measuring changes in the alternatively spliced region. Furthermore, specificity and efficiency for primers were analyzed by running RT-PCR with series of cDNA dilutions, and specific amplification for every assay was confirmed by melt curve analysis. The amplified transcripts were quantified using the comparative $\Delta \Delta \mathrm{Ct}$ method. GAPDH and HPRT were used as the internal control. SI was calculated for (A) by normalizing FC to the average FC of (C) for each splicing event. All assays were run in triplicates and were repeated three times. A raw $\mathrm{Ct}$ of 35 is used as the limit of detection: $\mathrm{Ct}$ values are set at 35 for any replicates with $\mathrm{Ct}$ values not determined or $>35$.

\section{Raw data processing, alignment analysis, and identification of differentially expressed genes and ASEs}

High-quality RNA samples were extracted, and illumine library was constructed as described earlier. Libraries were pooled and diluted for sequencing with a $1 \%$ PhiX spike-in according to Illumina protocol. The pool of library was loaded onto the HiSeq was performed using a 300-cycle high output v2 kit. Reads were obtained from sequencer or were downloaded from GEO. Adapter sequences and invalid reads containing poly- $\mathrm{N}$ and low-quality were removed using the FastX tool kit (v 0.0.14). The quality of reads was then confirmed using fastqc tool kit (v 0.11.5). All downstream analysis used the cleaned reads. The clean reads were mapped to the ENSEMBL built GRC38 using the STAR aligner (v2.5.3a) using ENCODE option as described in the STAR manual. Principal component analysis was performed to calculate variance within each group, and differential expression of genes was obtained using the DESeq2 (v1.28.0) (https://bioconductor.org/packa ges/release/bioc/html/DESeq2.html) method as described earlier [56]. Subsequently, we used rMATS (version3.0.8) (http://rnaseq-mats.sourceforge.net) to identify differentially ASE between the two sample groups. Briefly, rMATS uses a modified version of the generalized linear mixed model to detect differentially ASE from RNA-Seq data with replicates, while controlling for changes in expression at gene level. In addition, it also accounts for exon-specific sequencing coverage in individual samples as well as variation in exon splicing levels among replicates. rMATS was run using the default parameters and then significant splicing events were filtered using a stringent cutoff of FDR $\leq$ 0.05 and delta PSI $\geq 10 \%$. The Maser (v1.6.0) https://www. bioconductor.org/packages/release/bioc/html/maser.html was used for extracting and visualizing splice events.

\section{Motif enrichment analysis}

We employed rMAPS2 (http://rmaps.cecsresearch.org) analysis to determine the binding patterns of splicing factors and RNA binding proteins within significantly detected exon skipping ASE between two treatment groups. We collected well-characterized 115 known binding sites of RNA binding proteins. For each motif, the analysis scanned for motif occurrences separately in exons or their 250-bp upstream or downstream intronic sequences. Furthermore, for the intronic sequences our analysis excluded the 20-bp sequences within the $3^{\prime}$ splice site and the 6-bp sequences within the $5^{\prime}$ splice site. In addition, by default the alternative exons without splicing changes as defined by rMATS FDR $>50 \%$, maxPSI $>15 \%$, and $\operatorname{minPSI}<85 \%$ were treated as control exons. For each motif tested, the analysis counted the number of occurrences and motif score in the upstream exon, upstream flanking intron, target exon, downstream flanking intron, and downstream exon separately. The $p$ value for motif enrichment was calculated using the Wilcoxon's rank sum test for each sliding window between upregulated versus control or downregulated versus control exons.

\section{Functional annotation of DEGs and ASE}

ReactomePA (v1.32.0) and the clusterProfiler (v3.16.0) were used to generate lists of the GO terms enriched in the DEGs and ASE. The integration of protein features to splicing events was carried out using maser package. Briefly, maser enables systematic mapping of protein annotation from UniprotKB to splicing events and determine whether the splicing is affection regions of interest containing known domains or motifs, mutations, posttranslational modification, and other described protein structural features.

\section{Data availability}

Microarray Data: GSE150475.RNA-seq Data: SUB7404248.

\section{Code availability}

All the analysis was performed using vignette described for by the author of package on their github/website. The version and link to github/wesites for the tools and description on vignette used for analysis are mentioned in the "Material and method" section.

Acknowledgements This work was supported by grants from the intramural research program of the Division of Cancer Epidemiology and Genetics, National Cancer Institute, National Institutes of Health. 
This work utilized the computational resources of the NIH HPC Biowulf cluster and DCEG CCAD cluster (http://hpc.nih.gov).

\section{Compliance with ethical standards}

Conflict of interest The authors declare that they have no conflict of interest.

Publisher's note Springer Nature remains neutral with regard to jurisdictional claims in published maps and institutional affiliations.

Open Access This article is licensed under a Creative Commons Attribution 4.0 International License, which permits use, sharing, adaptation, distribution and reproduction in any medium or format, as long as you give appropriate credit to the original author(s) and the source, provide a link to the Creative Commons license, and indicate if changes were made. The images or other third party material in this article are included in the article's Creative Commons license, unless indicated otherwise in a credit line to the material. If material is not included in the article's Creative Commons license and your intended use is not permitted by statutory regulation or exceeds the permitted use, you will need to obtain permission directly from the copyright holder. To view a copy of this license, visit http://creativecommons. org/licenses/by/4.0/.

\section{References}

1. Huggins C. Prostatic cancer treated by orchiectomy; the five year results. J Am Med Assoc. 1946;131:576-81.

2. Azad AA, Zoubeidi A, Gleave ME, Chi KN. Targeting heat shock proteins in metastatic castration-resistant prostate cancer. Nat Rev Urol. 2015;12:26-36.

3. Georget V, Lobaccaro JM, Terouanne B, Mangeat P, Nicolas JC, Sultan C. Trafficking of the androgen receptor in living cells with fused green fluorescent protein-androgen receptor. Mol Cell Endocrinol. 1997;129:17-26.

4. Georget V, Terouanne B, Nicolas JC, Sultan C. Mechanism of antiandrogen action: key role of hsp90 in conformational change and transcriptional activity of the androgen receptor. Biochemistry. 2002;41:11824-31.

5. Shang Y, Myers M, Brown M. Formation of the androgen receptor transcription complex. Mol Cell. 2002;9:601-10.

6. Wang Q, Li W, Liu XS, Carroll JS, Janne OA, Keeton EK, et al. A hierarchical network of transcription factors governs androgen receptor-dependent prostate cancer growth. Mol Cell. 2007;27:380-92.

7. Wong CI, Zhou ZX, Sar M, Wilson EM. Steroid requirement for androgen receptor dimerization and DNA binding. Modulation by intramolecular interactions between the $\mathrm{NH} 2$-terminal and steroidbinding domains. J Biol Chem. 1993;268:19004-12.

8. Baniwal SK, Khalid O, Sir D, Buchanan G, Coetzee GA, Frenkel B. Repression of Runx 2 by androgen receptor (AR) in osteoblasts and prostate cancer cells: AR binds Runx2 and abrogates its recruitment to DNA. Mol Endocrinol. 2009;23:1203-14.

9. Grosse A, Bartsch S, Baniahmad A. Androgen receptor-mediated gene repression. Mol Cell Endocrinol. 2012;352:46-56.

10. Liu YN, Liu Y, Lee HJ, Hsu YH, Chen JH. Activated androgen receptor downregulates E-cadherin gene expression and promotes tumor metastasis. Mol Cell Biol. 2008;28:7096-108.

11. Prescott J, Jariwala U, Jia L, Cogan JP, Barski A, Pregizer S, et al. Androgen receptor-mediated repression of novel target genes. Prostate. 2007;67:1371-83.

12. Verras M, Lee J, Xue H, Li TH, Wang Y, Sun Z. The androgen receptor negatively regulates the expression of c-Met: implications for a novel mechanism of prostate cancer progression. Cancer Res. 2007;67:967-75

13. Zhang Y, Pitchiaya S, Cieslik M, Niknafs YS, Tien JC, Hosono Y, et al. Analysis of the androgen receptor-regulated lncRNA landscape identifies a role for ARLNC1 in prostate cancer progression. Nat Genet. 2018;50:814-24.

14. Huang H, Tindall DJ. The role of the androgen receptor in prostate cancer. Crit Rev Eukaryot Gene Expr. 2002;12:193-207.

15. Chen CD, Welsbie DS, Tran C, Baek SH, Chen R, Vessella R, et al. Molecular determinants of resistance to antiandrogen therapy. Nat Med. 2004;10:33-9.

16. Lu-Yao GL, Albertsen PC, Moore DF, Shih W, Lin Y, DiPaola RS, et al. Fifteen-year survival outcomes following primary androgen-deprivation therapy for localized prostate cancer. JAMA Intern Med. 2014;174:1460-7.

17. Watson PA, Arora VK, Sawyers CL. Emerging mechanisms of resistance to androgen receptor inhibitors in prostate cancer. Nat Rev Cancer. 2015;15:701-11.

18. Wong YN, Ferraldeschi R, Attard G, de Bono J. Evolution of androgen receptor targeted therapy for advanced prostate cancer. Nat Rev Clin Oncol. 2014;11:365-76.

19. Sirotnak FM, She Y, Khokhar NZ, Hayes P, Gerald W, Scher HI. Microarray analysis of prostate cancer progression to reduced androgen dependence: studies in unique models contrasts early and late molecular events. Mol Carcinog. 2004;41:150-63.

20. Chen S, Xu Y, Yuan X, Bubley GJ, Balk SP. Androgen receptor phosphorylation and stabilization in prostate cancer by cyclindependent kinase 1. Proc Natl Acad Sci USA. 2006;103: 15969-74.

21. Gregory CW, Fei X, Ponguta LA, He B, Bill HM, French FS, et al. Epidermal growth factor increases coactivation of the androgen receptor in recurrent prostate cancer. J Biol Chem. 2004;279:7119-30.

22. Gregory CW, Whang YE, McCall W, Fei X, Liu Y, Ponguta LA, et al. Heregulin-induced activation of HER2 and HER3 increases androgen receptor transactivation and CWR-R1 human recurrent prostate cancer cell growth. Clin Cancer Res. 2005;11:1704-12.

23. Mellinghoff IK, Vivanco I, Kwon A, Tran C, Wongvipat J, Sawyers CL. HER2/neu kinase-dependent modulation of androgen receptor function through effects on DNA binding and stability. Cancer Cell. 2004;6:517-27.

24. Weber MJ, Gioeli D. Ras signaling in prostate cancer progression. J Cell Biochem. 2004;91:13-25.

25. Hammes A, Andreassen TK, Spoelgen R, Raila J, Hubner N, Schulz $\mathrm{H}$, et al. Role of endocytosis in cellular uptake of sex steroids. Cell. 2005;122:751-62.

26. Fizazi K, Scher HI, Molina A, Logothetis CJ, Chi KN, Jones RJ, et al. Abiraterone acetate for treatment of metastatic castrationresistant prostate cancer: final overall survival analysis of the COU-AA-301 randomised, double-blind, placebo-controlled phase 3 study. Lancet Oncol. 2012;13:983-92.

27. Furr BJ, Valcaccia B, Curry B, Woodburn JR, Chesterson G, Tucker H. ICI 176,334: a novel non-steroidal, peripherally selective antiandrogen. J Endocrinol. 1987;113:R7-9.

28. Tran C, Ouk S, Clegg NJ, Chen Y, Watson PA, Arora V, et al. Development of a second-generation antiandrogen for treatment of advanced prostate cancer. Science. 2009;324:787-90.

29. Penson DF, Armstrong AJ, Concepcion R, Agarwal N, Olsson C, Karsh L, et al. Enzalutamide versus bicalutamide in castrationresistant prostate cancer: the STRIVE Trial. J Clin Oncol. 2016;34:2098-106.

30. Lee Y, Rio DC. Mechanisms and regulation of alternative PremRNA splicing. Annu Rev Biochem. 2015;84:291-323.

31. Nilsen TW, Graveley BR. Expansion of the eukaryotic proteome by alternative splicing. Nature. 2010;463:457-63. 
32. David CJ, Manley JL. Alternative pre-mRNA splicing regulation in cancer: pathways and programs unhinged. Genes Dev. 2010;24:2343-64.

33. Kahles A, Lehmann KV, Toussaint NC, Huser M, Stark SG, Sachsenberg T, et al. Comprehensive analysis of alternative splicing across tumors from 8,705 patients. Cancer Cell. 2018;34:211-24.e6.

34. Auboeuf D, Honig A, Berget SM, O'Malley BW. Coordinate regulation of transcription and splicing by steroid receptor coregulators. Science. 2002;298:416-9.

35. Dutertre M, Gratadou L, Dardenne E, Germann S, Samaan S, Lidereau R, et al. Estrogen regulation and physiopathologic significance of alternative promoters in breast cancer. Cancer Res. 2010;70:3760-70.

36. Braunschweig U, Barbosa-Morais NL, Pan Q, Nachman EN, Alipanahi B, Gonatopoulos-Pournatzis T, et al. Widespread intron retention in mammals functionally tunes transcriptomes. Genome Res. 2014;24:1774-86.

37. Dvinge H, Bradley RK. Widespread intron retention diversifies most cancer transcriptomes. Genome Med. 2015;7:45.

38. Jung H, Lee D, Lee J, Park D, Kim YJ, Park WY, et al. Intron retention is a widespread mechanism of tumor-suppressor inactivation. Nat Genet. 2015;47:1242-8.

39. Molenaar RJ, Maciejewski JP, Wilmink JW, van Noorden CJF. Wild-type and mutated IDH1/2 enzymes and therapy responses. Oncogene. 2018;37:1949-60.

40. Rasmussen KD, Helin K. Role of TET enzymes in DNA methylation, development, and cancer. Genes Dev. 2016;30: 733-50

41. Park JW, Jung S, Rouchka EC, Tseng YT, Xing Y. rMAPS: RNA map analysis and plotting server for alternative exon regulation. Nucleic Acids Res. 2016;44:W333-338.

42. Yang Y, Park JW, Bebee TW, Warzecha CC, Guo Y, Shang X, et al. Determination of a comprehensive alternative splicing regulatory network and combinatorial regulation by key factors during the epithelial-to-mesenchymal transition. Mol Cell Biol. 2016;36:1704-19.

43. Munkley J, Li L, Krishnan SRG, Hysenaj G, Scott E, Dalgliesh C, et al. Androgen-regulated transcription of ESRP2 drives alternative splicing patterns in prostate cancer. Elife. 2019;8:e47678.

44. Warzecha CC, Jiang P, Amirikian K, Dittmar KA, Lu H, Shen S, et al. An ESRP-regulated splicing programme is abrogated during the epithelial-mesenchymal transition. EMBO J. 2010;29: 3286-300.
45. Lin TH, Lee SO, Niu Y, Xu D, Liang L, Li L, et al. Differential androgen deprivation therapies with anti-androgens casodex/ bicalutamide or MDV3100/Enzalutamide versus anti-androgen receptor ASC-J9(R) Lead to promotion versus suppression of prostate cancer metastasis. J Biol Chem. 2013;288:19359-69.

46. Yun SJ, Kim SK, Kim J, Cha EJ, Kim JS, Kim SJ, et al. Transcriptomic features of primary prostate cancer and their prognostic relevance to castration-resistant prostate cancer. Oncotarget. 2017;8:114845-55.

47. Ganesan K, Ivanova T, Wu Y, Rajasegaran V, Wu J, Lee MH, et al. Inhibition of gastric cancer invasion and metastasis by PLA2G2A, a novel beta-catenin/TCF target gene. Cancer Res. 2008;68:4277-86.

48. Olivier JL, Fan Q, Salvat C, Ziari M, Kong L, Mangeney M, et al. Positive and negative hepatic regulation of the human type II phospholipase A2 gene. Biochemistry. 1994;33:7134-45.

49. Paradon M, Salvat C, et al. An SP1-like 5'-GACCACGCC-3' sequence is critical for activity of the inflammatory phospholipase A2 promoter and binds several non-zinc finger proteins. Eur J Biochem. 1998;258:113-22.

50. Di C, Syafrizayanti, Zhang Q, Chen Y, Wang Y, Zhang X, et al. Function, clinical application, and strategies of Pre-mRNA splicing in cancer. Cell Death Differ. 2019;26:1181-94.

51. Miao L, Yang L, Li R, Rodrigues DN, Crespo M, Hsieh JT, et al. Disrupting androgen receptor signaling induces snail-mediated epithelial-mesenchymal plasticity in prostate cancer. Cancer Res. 2017;77:3101-12.

52. Stylianou N, Lehman ML, Wang C, Fard AT, Rockstroh A, Fazli $\mathrm{L}$, et al. A molecular portrait of epithelial-mesenchymal plasticity in prostate cancer associated with clinical outcome. Oncogene. 2019;38:913-34.

53. Smart AC, Margolis CA, Pimentel H, He MX, Miao D, Adeegbe $\mathrm{D}$, et al. Intron retention is a source of neoepitopes in cancer. Nat Biotechnol. 2018;36:1056-8.

54. Iwasaki K, Ninomiya R, Shin T, Nomura T, Kajiwara T, Hijiya N, et al. Chronic hypoxia-induced slug promotes invasive behavior of prostate cancer cells by activating expression of ephrin-B1. Cancer Sci. 2018;109:3159-70.

55. Liu W, Wang X, Wang Y, Dai Y, Xie Y, Ping Y, et al. SGK1 inhibition-induced autophagy impairs prostate cancer metastasis by reversing EMT. J Exp Clin Cancer Res. 2018;37:73.

56. Stebbing J, Shah K, Lit LC, Gagliano T, Ditsiou A, Wang T, et al. LMTK3 confers chemo-resistance in breast cancer. Oncogene. 2018;37:3113-30. 